\title{
Research Paper \\ Development of Nurses' Beliefs and Attitudes Towards Complementary and Alternative Medicine Questionnaire and its Psychometric Evaluation
}

\author{
Nafiseh Kaviani ${ }^{1} \odot$, *Zahra Tagharrobi ${ }^{1} \odot$, Khadijeh Sharifi ${ }^{1} \odot$, Zahra Sooki ${ }^{1} \odot$
}

1. Trauma Nursing Research Centre, Faculty of Nursing and Midwifery, Kashan University of Medical Sciences, Kashan, Iran.

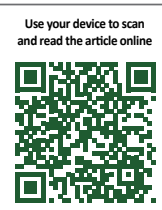

Citation: Kaviani N, Tagharrobi Z, Sharifi Kh, Sooki Z. [Development and Psychometric Evaluation of Iranian Nurses' Beliefs and Attitudes Toward Complementary Therapies Inventory Based on the Theory of Planned Behavior (Persian)]. Complementary Medicine Journal. 2020; 10(1):12-33. https://doi.org/10.32598/cmja.10.1.589.2

https://doi.org/10.32598/cmja.10.1.589.2

Keywords:

Complementary alternative medicine, Attitudes, Psychometrics, Nursing, Theory of planned behavior

\section{ABSTRACT}

Objective Due to the impact of the beliefs and attitudes on clinical practice, it is necessary to measure the beliefs and attitudes of nurses towrads the use of Complementary and Alternative Medicine (CAM) in patient care. This study aimed for development and psychometric evaluation of Nurses' Beliefs and Attitudes towards CAM Questionnaire (NBACQ) for Iranian population.

Methods In this methodological study, based on Waltz et al.'s approach and the constructs of the theory of planned behavior, the initial version of NBACQ was designed, and its face and content validity were assessed. Participants were 250 eligible clinical nurses in Kashan, Iran selected using a stratified random sampling method in 2018 after obtaining informed written consent from them. Construct validity of the tool was evaluated by factor analysis and known-groups method, and its reliability was assessed by measuring its internal consistency and stability as well as the ceiling and floor effects. Data were analyzed in SPSS by using exploratory factor analysis, ANOVA, Cronbach's alpha and Intraclass Correlation Coefficient (ICC).

Results The initial version of NBACQ had 60 items. In the face and content validity assessment stage, 4 items were eliminated. Factor analysis yielded 6 factors by eliminating 14 items (Explained Variance $=52.3 \%$ ). There was a significant relationship between the NBACQ score and the nurses' agreement with the use of CAM in clinical practice $(F=6.85, P<0.0001)$. Cronbach's alpha and ICC values were obtained 0.878 and 0.973 , respectively. The standard error of measurement and the smallest detectable change were calculated \pm 14.685 and 10.62 , respectively. The frequency of minimum and maximum possible NBACQ scores was zero.

Conclusion The 42-item NBACQ can be used as a valid and reliable tool for assessing the nurses' beliefs and attitudes towards CAM.

\section{Extended Abstract}

\section{Introduction}

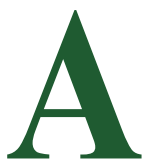

mong the possible influencing factors, the role of nurses' beliefs and attitudes towards Complementary and Alternative Medicine (CAM) has been emphasized $[15,16]$. In order to measure the belief and attitude of the treatment staff in Iranian society and culture, it is necessary to have a suitable tool. Based on the data recorded in the available national and international databases, a questionnaire that can covers different dimensions of belief and attitude and be specific to measuring the attitude and attitude of nursing staff towards CAM, has not yet been designed, localized, and psychometrically evaluated. Therefore, this study

* Corresponding Author:

Zahra Tagharrobi, PhD.

Address: Trauma Nursing Research Centre, Faculty of Nursing and Midwifery, Kashan University of Medical Sciences, Kashan, Iran.

Tel: +98 (913) 1613899

E-mail: tagharrobi_z@yahoo.com 
aimed to design a questionnaire for measuring belief and attitude towards the CAM for nurses in Iran working in the clinical fields, based on the constructs proposed in the theory of planned behavior, and evaluate its psychometric properties.

\section{Materials and Methods}

In this methodological study, first the initial version of the Nurses' Beliefs and Attitudes towards CAM Questionnaire (NBACQ) was developed based on the 4-step approach of Waltz et al. [31] by reviewing the studies and based on the theory of planned behavior. The second phase was related to psychometric evaluation in three steps: 1 . measuring face and content validity; 2 . measuring construct validity (exploratory factor analysis and comparison of known groups) and ceiling and floor effect; and 3. assessing the reliability (internal consistency and stability) [33].

In order to perform the second step of the phase 2 and determine the internal consistency of the NBACQ, 250 eligible nurses were selected using stratified sampling method in June 2018 from different wards in hospitals affiliated to Kashan University of Medical Sciences, and then were asked to complete the initial version of the NBACQ. To measure the stability of the tool, the test-retest method was performed on 20 samples [33, 34].

Collected data were analyzed in SPSS V. 16. Content Validity Index (CVI), Content Validity Ratio (CVR), and modified kappa statistic were used for measuring the content validity, item impact test for measuring the face validity, exploratory factor analysis for measuring construct validity, One-way ANOVA to compare known groups, Cronbach's alpha coefficient to check the internal consistency, and Intraclass Correlation Coefficient (ICC) for assessing the correlation of scores reported by two raters in the test-retest method. Standard Error of Measurement (SEM) and Smallest Detectable Change (SDC) were also calculated [34].

\section{Results}

The initial draft of NBACQ had 60 items. CVRStrict was three items lower than that reported by Ayre \& Scally (2014); hence, these three items were removed. The calculated CVI for each item was in the range of 0.8-1. The

Table 1. Exploratory factor analysis results of the final version of NBACQ

\begin{tabular}{ccc}
\hline Factor & Eigen Value & Explained Variance (\%) \\
\hline $1^{\text {st }}$ factor (16 items) & 10.34 & 18.47 \\
$2^{\text {nd }}$ factor (6 items) & 4.24 & 7.58 \\
\hline $3^{\text {rd }}$ factor (6 items) & 3.97 & 7.10 \\
$4^{\text {th }}$ factor (4 items) & 3.78 & 6.74 \\
$5^{\text {th }}$ factor (7 items) & 3.73 & 6.67 \\
$6^{\text {th }}$ factor (3 items) & 3.21 & 5.73 \\
Total & & 52.28 \\
\hline
\end{tabular}

Table 2. Mean NBACQ score - attitude towards CAM ( $\mathrm{n}=250)$

\begin{tabular}{|c|c|c|c|c|c|c|c|c|}
\hline \multirow{4}{*}{ NBACQ } & \multicolumn{7}{|c|}{ Attitude Towards CAM (1 item) } & \multirow{4}{*}{$\begin{array}{c}\text { One-way } \\
\text { ANOVA Result }\end{array}$} \\
\hline & 1 & 2 & 3 & 4 & 5 & 6 & 7 & \\
\hline & $\begin{array}{l}\text { Complete- } \\
\text { ly Disagree }\end{array}$ & Disagree & $\begin{array}{c}\text { Somewhat } \\
\text { Disagree }\end{array}$ & Neutral & $\begin{array}{l}\text { Somewhat } \\
\text { Agree }\end{array}$ & Agree & $\begin{array}{l}\text { Complete- } \\
\text { ly Agree }\end{array}$ & \\
\hline & $(n=1)$ & $(n=4)$ & $(n=8)$ & $(n=24)$ & $(n=75)$ & $(n=98)$ & $(n=40)$ & \\
\hline $\begin{array}{c}\text { Score } \\
\text { (mean } \pm S D)\end{array}$ & 185 & $\begin{array}{c}162.50 \pm \\
29.352\end{array}$ & $\begin{array}{c}156.62 \pm \\
20.37\end{array}$ & $\begin{array}{c}174.620 \pm \\
14.68\end{array}$ & $\begin{array}{c}172.51 \pm \\
19.40\end{array}$ & $\begin{array}{c}183.66 \pm \\
17.96\end{array}$ & $\begin{array}{c}187.18 \pm \\
25.90\end{array}$ & $\begin{array}{rl} & F=6.58 \\
* & P<0.0001\end{array}$ \\
\hline
\end{tabular}

Note: to perform post-hoc test, group 1 (completely disagree) was removed due to having one sample;* significant difference in attitude scores between groups 3 and $6(\mathrm{P}<0.0001)$, between groups 3 and $7(\mathrm{P}<0.0001)$, between groups 5 and $6(\mathrm{P}=0.004)$, and between groups 5 and $7(\mathrm{P}=0.003)$ 
kappa statistic of all items was above 0.74 and the S-CVIAverage of the tool was obtained 0.97 .

The item impact of all items was calculated to be above 1.5 , except for one item which was removed. Exploratory factor analysis yielded 6 factors in the tool by removing 14 items. These factors were named as "patient-centered outcomes", "normative beliefs", "executive needs", "treatment team performance", "infrastructural beliefs" and "professional outcomes" which could explain $52.28 \%$ of total variance (Table 1, and Appendix 1).

Based on the scores of designed 42-item NBACQ, the overall score of nurses' beliefs and attitudes (on a scale from 42 to 294 ) was $178.81 \pm 20.948$, and their score based on the response to the 1-item attitude assessment was $5.488 \pm 1.109$ (on a scale of 1-7). There was a statistically significant difference between the scores of nurses' beliefs and attitudes in terms of their degree of agreement or disagreement with $\mathrm{CAM}(\mathrm{F}=6.85, \mathrm{P}<0.0001)$ (Table 2). Cronbach's alpha coefficient for the overall reliability of the NBACQ was calculated to be 0.878 , and the ICC between test-retest scores for the whole instrument was reported 0.973 (CI: 0.932-0.989, $\mathrm{P}<0.0001)$. Furthermore, SEM and SDC were obtained \pm 14.685 and 10.62 , respectively at $95 \%$ confidence interval.

\section{Discussion}

Due to the use of theoretical infrastructure in designing the tool $[31,32]$ in this study, and the values of CVI, CVR, S-CVIAverage and kappa statistic, it can be said that the NBACQ had met the necessary criteria for content validity [33]. Based on the impact score of all remained items, it was found that there was no problem in perceiving the NBACQ items by the target group and, hence, its face validity is confirmed [34]. Having 6 factors indicates the tools' optimal construct [34] and shows that there is compatibility between these factors and the belief and attitude concepts in the theory of planned behavior. The construct of this tool was more desirable than that of similar tools [20, 21]; Of course, examining its construct using confirmatory factor analysis provides more accurate information. The result of comparing known groups was also in favor of the construct validity of the instrument [39].

The absence of ceiling and floor effects indicated the adequacy and appropriateness of the used items and supported the content validity and stability of the NBACQ [36]. The Cronbach's alpha coefficient and the ICC of the whole instrument indicated the desired its internal consistency [31] and stability [33], respectively, and showed that this NBACQ has a higher internal consistency compared to Integrative
Medicine Attitude Questionnaire (IMAQ) and CAM Health Belief Questionnaire (CHBQ) [21, 22].

\section{Conclusion}

The study showed that if the test be repeated for an individual, the score may change as 14.68 units. Given that the small size of the SEM is important and indicates the stability of the tool [34], its stability, repeatability and in overall, reliability, can be considered desirable. Therefore, it can be concluded that the 42-item NBACQ designed in this study is a valid and reliable tool and can be used to measure nurses' beliefs and attitudes toward CAM. It is suggested that its short form be designed in future studies.

\section{Ethical Considerations}

\section{Compliance with ethical guidelines}

This study obtained ethical approval from the Research Ethics Committee of Kashan University of Medical Sciences (Code: IR.KAUMS.NUHEPM.REC.1396.35).

\section{Funding}

This study was extracted from the master thesis of the first author approved by Kashan University of Medical Sciences and received financial support from the Deputy for Research and Technology of this university (Grant no. 96225).

\section{Authors' contributions}

Conceptualization: All authors; data collection and initial draft preparation: Nafiseh Kabiani; data analysis, editing \& review, supervision: Zahra Tagharrobi, Khadihej Sharifi, and Zahra Sooki.

\section{Conflicts of interest}

The authors declare no conflict of interest

\section{Acknowledgements}

The authors would like to thank the participants and the Deputy for Research and Technology of Kashan University of Medical Sciences for their cooperation and support. 
Appendix 1. The items of the initial version of NBACQ (in English) and their factor load

\begin{tabular}{|c|c|c|c|c|c|c|}
\hline \multirow[t]{2}{*}{ Item } & \multicolumn{6}{|c|}{ Extracted Factors } \\
\hline & 1 & 2 & 3 & 4 & 5 & 6 \\
\hline $\begin{array}{c}\text { CAM in the form of nursing care helps reduce patients' physical } \\
\text { problems }\end{array}$ & 0.73 & & & & & \\
\hline $\begin{array}{c}\text { CAM in the form of nursing care helps reduce patients' psychological } \\
\text { problems }\end{array}$ & 0.77 & & & & & \\
\hline
\end{tabular}

CAM helps instill a sense of wellness in patients

CAM helps terminally-ill patients die in peace

CAM helps patients increase motivation to get better

CAM helps difficult-to-treat patients accept the disease better and follow the treatment process

0.77

0.53

0.77

0.72

CAM helps gain the cooperation from the patient and build a trustbased relationship between the patient and the nurse.

CAM creates a sense of relaxation in patients (and their family)

The use of CAM in the form of nursing care has been scientifically proven

CAM along with other conventional methods shortens the course of treatment and speeds up the healing process.

CAM reduces the length of stay in hospital

CAM is effective in preventing some physical problems in patients.

CAM is effective in preventing some psychological problems in patients

If the patient's problems do not improve with conventional treatment methods, nurses should try CAM

The use of CAM in the form of nursing care is safe for patients

CAM is more effective than conventional medicine

The use of CAM in clinical practice helps the professional independence of the nurse

The use of CAM in clinical practice helps introduce the nurse as a professional

CAM is a threat to public health

CAM has fewer side effects than conventional medicine

The use of CAM in the form of nursing care increases the patient's satisfaction with the provided services

The effects of CAM are no more than those of placebo 


\begin{tabular}{|c|c|c|c|c|c|c|}
\hline \multirow[t]{2}{*}{ Item } & \multicolumn{6}{|c|}{ Extracted Factors } \\
\hline & 1 & 2 & 3 & 4 & 5 & 6 \\
\hline $\begin{array}{l}\text { The use of CAM in the form of nursing care builds trust between } \\
\text { physicians and nurses }\end{array}$ & & & & & & 0.57 \\
\hline \multicolumn{7}{|l|}{$\begin{array}{l}\text { The use of CAM in clinical practice creates positive emotions (calm- } \\
\text { ness, satisfaction, pleasure, self-confidence, etc.) in the nurse }\end{array}$} \\
\hline \multicolumn{7}{|l|}{$\begin{array}{l}\text { I believe nurses should try the CAM methods recommended by physi- } \\
\text { cians in their clinical practice and observe the results }\end{array}$} \\
\hline $\begin{array}{l}\text { I believe nurses should try the CAM methods recommended by col- } \\
\text { leagues in their clinical practice and observe the results }\end{array}$ & & 0.67 & & & & \\
\hline $\begin{array}{c}\text { I believe nurses should try the CAM methods recommended by mass } \\
\text { media (TV, radio, newspaper, social network, etc.) in their clinical } \\
\text { practice and observe the results }\end{array}$ & & 0.63 & & & & \\
\hline $\begin{array}{l}\text { I believe nurses should try the CAM methods tested by family mem- } \\
\text { bers and relatives in their clinical practice and observe the results }\end{array}$ & & 0.64 & & & & \\
\hline $\begin{array}{l}\text { I believe nurses should try the CAM methods recommended and } \\
\text { tested by nursing managers in their clinical practice and observe the } \\
\text { results }\end{array}$ & & 0.76 & & & & \\
\hline $\begin{array}{l}\text { I believe nurses should try the CAM methods recommended by } \\
\text { educated people and specialists in the field of CAM and introduced in } \\
\text { scientific journals or conferences, in their clinical practice and observe } \\
\text { the results }\end{array}$ & & 0.63 & & & & \\
\hline \multicolumn{7}{|l|}{$\begin{array}{c}\text { I believe in the effectiveness of CAM methods recommended in } \\
\text { religious narratives and hadiths }\end{array}$} \\
\hline $\begin{array}{c}\text { The professional nature of nursing requires nurses to use CAM in } \\
\text { patient care }\end{array}$ & & 0.61 & & & & \\
\hline
\end{tabular}

Morally, nurses are required to use CAM in patient care as much as possible to prevent the complications of taking high doses of medications

Nurses should be able to advise patients on the proper use of CAM.

The use of CAM in patient care is a simple task for clinical nurses and does not require much skill

The nurse does not need to spend a lot of time to use CAM in patient care

The nurse does not need specialized and complicated information

The nurse does not need a doctor's prescription to use CAM in patient care

No specific location or space is required for the use of CAM in patient care

A nurse who wants to use CAM in patient care should be fully aware of the effects, complications, and management of its side effects, regardless of how it is used.

Failure to set specific tariffs for CAM prevents it from being used in patient care

Not considering CAM in nurses' job descriptions prevents it from being used in patient care. 


\begin{tabular}{|c|c|c|c|c|c|c|}
\hline \multirow[t]{2}{*}{ Item } & \multicolumn{6}{|c|}{ Extracted Factors } \\
\hline & 1 & 2 & 3 & 4 & 5 & 6 \\
\hline $\begin{array}{l}\text { Not considering CAM in insurance coverage prevents it from being } \\
\text { used in patient care }\end{array}$ & & & & & 0.58 & \\
\hline \multicolumn{7}{|l|}{$\begin{array}{l}\text { It is difficult to attract the cooperation of the patient or his/her family } \\
\text { to help the nurse use CAM in patient care }\end{array}$} \\
\hline $\begin{array}{l}\text { It is difficult to attract the cooperation of authorities on using CAM in } \\
\text { patient care }\end{array}$ & & & & 0.83 & & \\
\hline $\begin{array}{l}\text { It is difficult to attract the cooperation of other colleagues to continue } \\
\text { to use CAM in other shifts }\end{array}$ & & & & 0.85 & & \\
\hline $\begin{array}{l}\text { It is difficult to attract the cooperation of staff to help the nurse use } \\
\text { CAM in patient care. }\end{array}$ & & & & 0.84 & & \\
\hline $\begin{array}{l}\text { It is difficult to attract the cooperation of physicians on using CAM in } \\
\text { patient care }\end{array}$ & & & & 0.76 & & \\
\hline $\begin{array}{l}\text { Further research is needed to ensure the safety and effectiveness of } \\
\qquad \text { CAM in patient care }\end{array}$ & & & & & 0.55 & \\
\hline \multicolumn{7}{|l|}{$\begin{array}{l}\text { Nursing education and management staff should try to link CAM to } \\
\text { the performance of clinical nurses }\end{array}$} \\
\hline Nurses need permission to use CAM in their clinical practice & & & & & 0.57 & \\
\hline \multicolumn{7}{|l|}{$\begin{array}{l}\text { Access to reputable and appropriate resources in the field of using } \\
\text { CAM in clinical nursing is difficult }\end{array}$} \\
\hline $\begin{array}{l}\text { Insufficient psychological preparation of the nurse (e.g. lack of con- } \\
\text { centration, impatience, fear, etc.) prevents the use of CAM in patient } \\
\text { care }\end{array}$ & & & & & 0.52 & \\
\hline $\begin{array}{l}\text { Crowded wards and the high volume of nursing tasks prevent the use } \\
\text { of CAM in clinical practice }\end{array}$ & & & & & 0.50 & \\
\hline
\end{tabular}

Note $=$ The minimum factor load is 0.5 ; factors $<0.5$ are not listed in the table; $*$ There was no common factor load;

Factor 1 (items 1-13, 15, 16, 21)= Patient-centered outcomes; factor 2 (items 26-30, 32) = Normative beliefs; factor 3 (items 35-40) $=$ Executive needs; factor $4($ items $47-50)=$ Treatment team performance; factor $5($ items $41,42,45,51,53,55,56)=$ Infrastructural beliefs; factor 6 (items 17, 18, 23) = Professional outcomes 
This Page Intentionally Left Blank 


\title{
طراحى و روانسنجى ابزار سنجش باور و نتَرش يرستاران نسبت به درمانهاى مكمل بر اساس

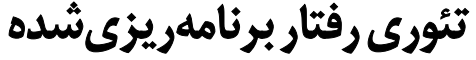

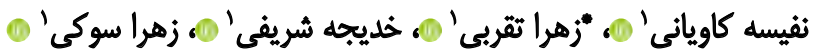

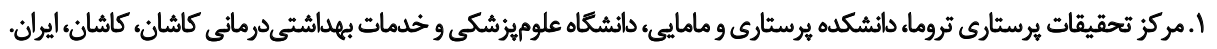

\begin{abstract}
אيكيد

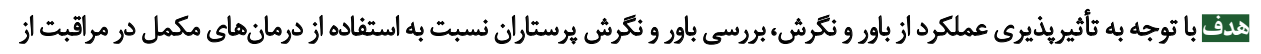

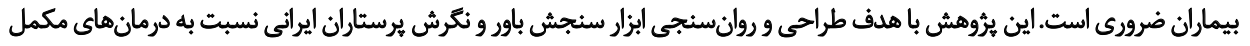

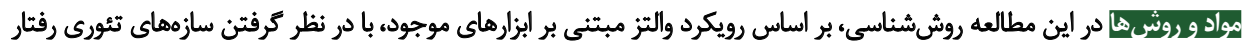

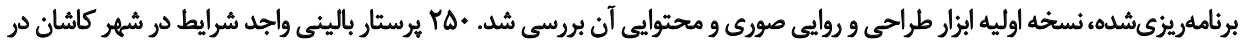

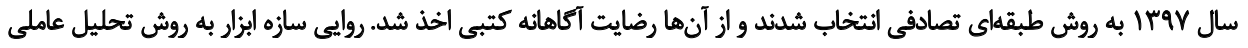

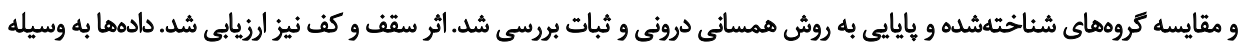

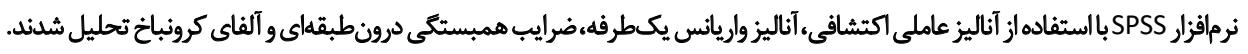

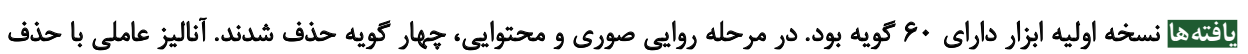
fif

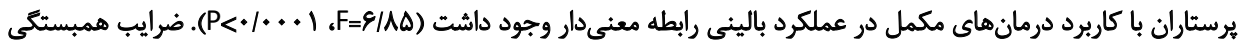

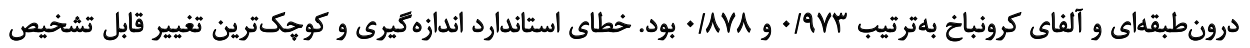

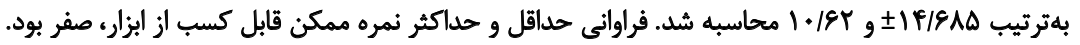

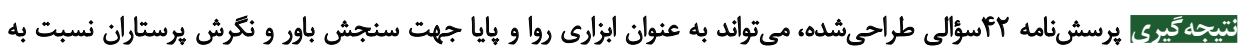
درمانهاي مكمل مورد استفاده قرار كيرد.
\end{abstract}

اطلاعات مقاله:

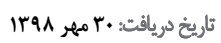

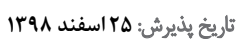
تاريخ انتشار: rاخرداد

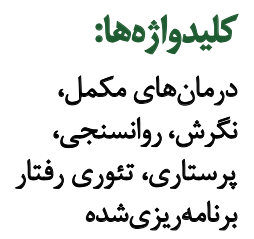

صرف نظر از عموم جمعيث، درصد قابل توجهى از بيماران

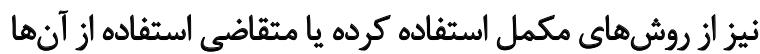

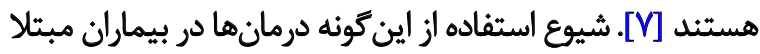

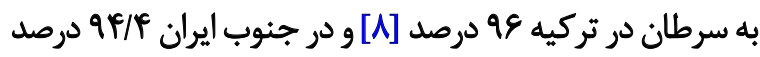

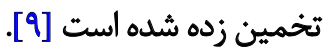

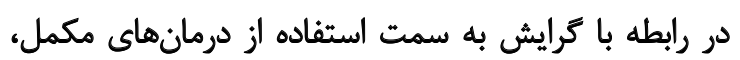

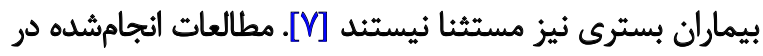

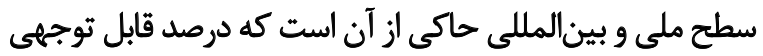

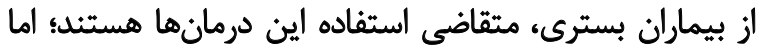

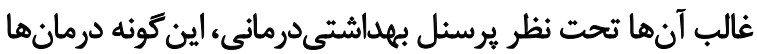

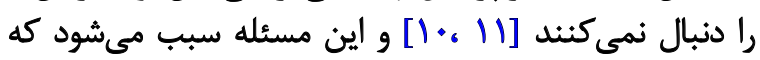

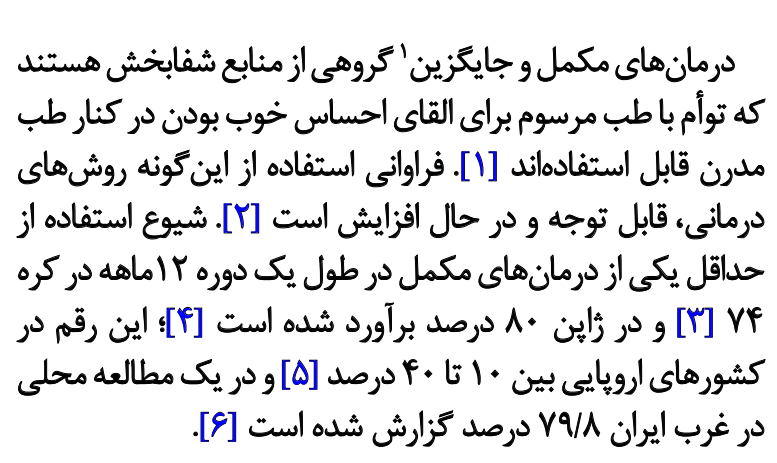

1. Complementary and Alternative Medicine (CAM) 


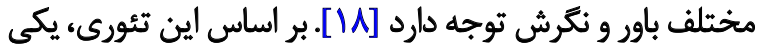

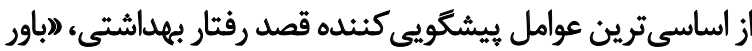

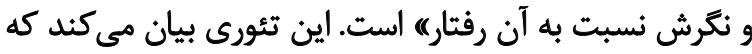

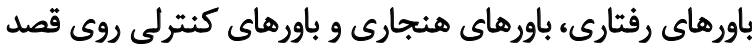

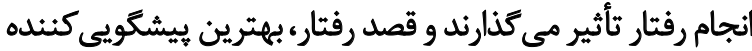

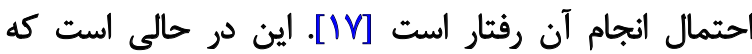

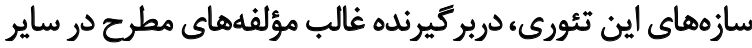

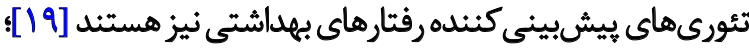
مورد اخير نيز از نقاط قوت تئورى موردنظر است.

مطالعات كوياى آن است كه تاكنون جندين ابزار جهت سنجش

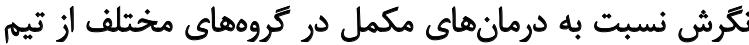

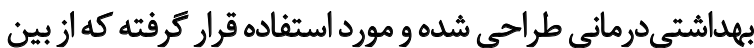
آنها مى توان به ابزار

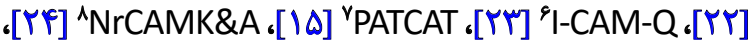

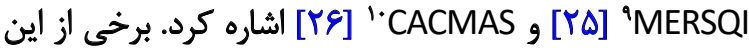

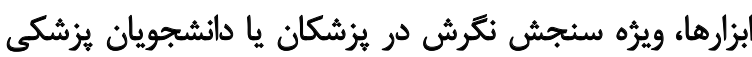

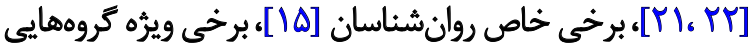

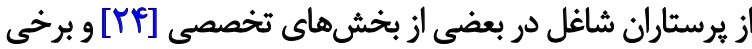

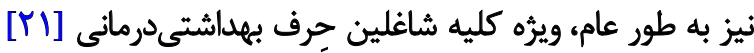

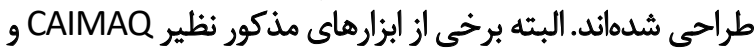

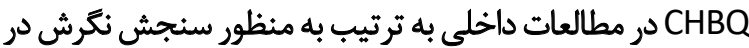

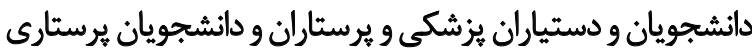

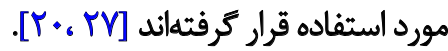
در ابزارهاي مورداستفادهر مطالعات بينالمللى،بيشتر به فلسفه

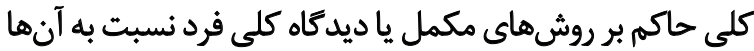

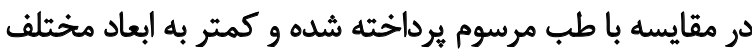

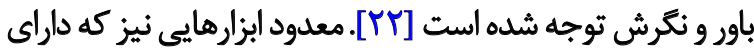

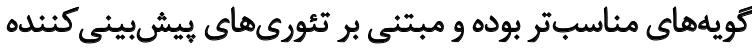

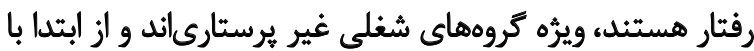

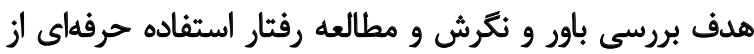

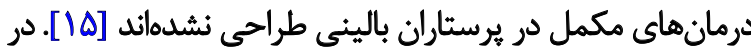

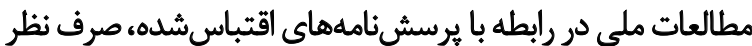

3. Complementary, Alternative and Integrative Medicine Attitude Questionnaire

4. Integrative Medicine Attitude Questionnaire

5. CAM Health Belief Questionnaire

6. International CAM Questionnaire

7. Psychologists Attitudes Towards Complementary and Alternative Therapies

8. Nurse Complementary and Alternative Medicine Knowledge and Attitude

9. Medical Education Research Study Quality Instrument

10. Complementary, Alternative and Conventional Medicine Attitudes Scale
درصد زيادى از آنها در دام افراد سودجو ترفتار شده و يا مسائل

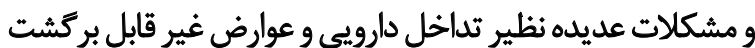

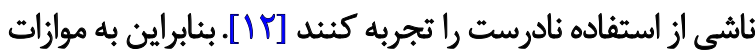

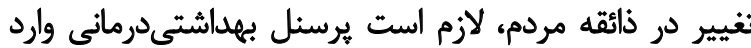

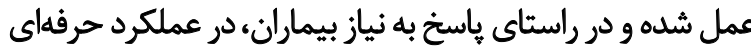
خويش از اين كونه روشهاي بهره كيرند

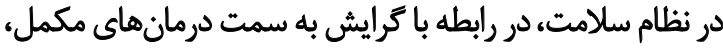

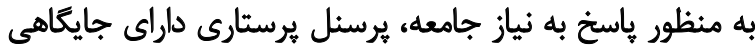

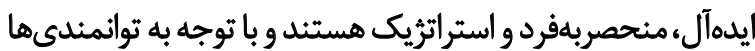

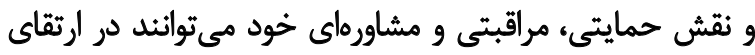

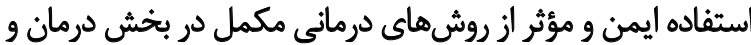

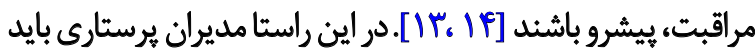

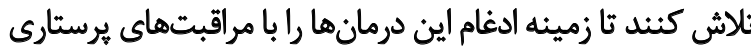

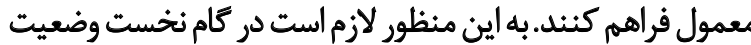

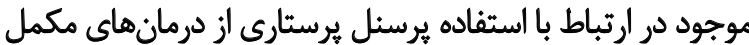
در عملكرد بالينى و عوامل مؤثر بر آن ارزيابى شود.

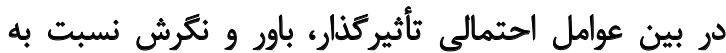

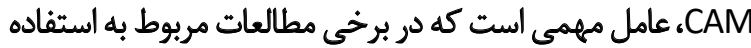

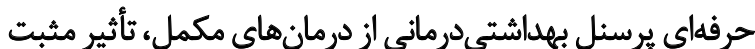

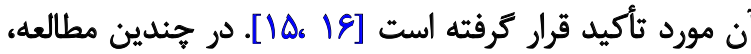

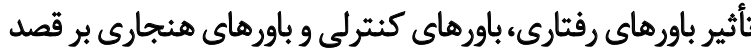

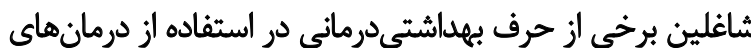

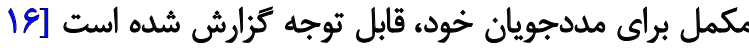

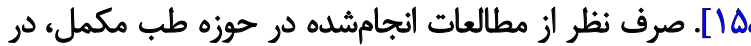

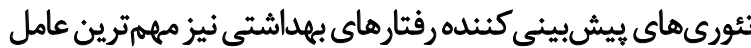

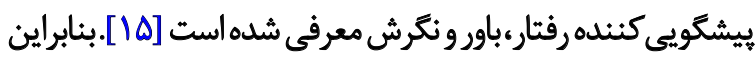

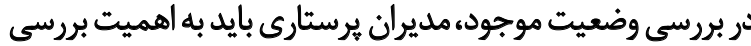
باور ونكرش يرستاران نسبت به CAM واقف باشئد.

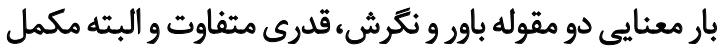

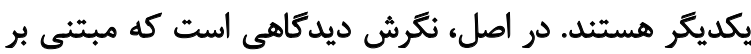

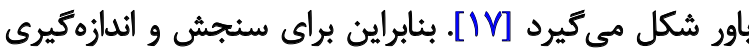

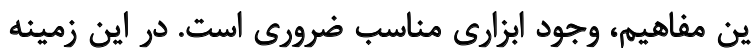

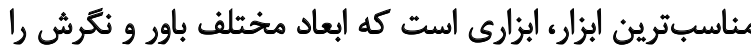

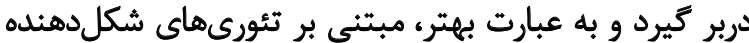

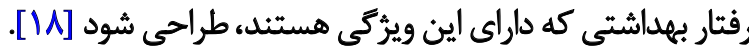

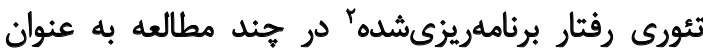

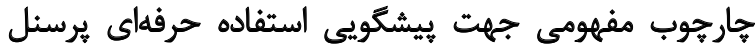

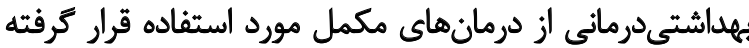

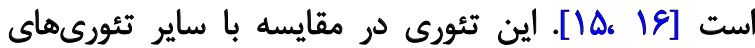

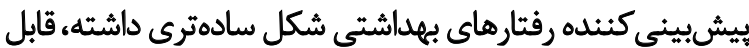
فههمتر و درعين حال جامعتر بوده و به محدوده وسيعترى از إبعاد

2. Theory of Planned Behavior (TPB) 
مرحله سوم، ييشنويس اوليه ابزار تدوين شد؛ يعنى كليه مقالات

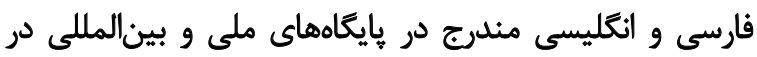

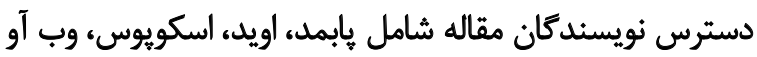

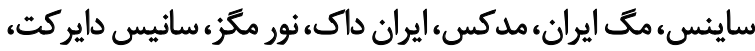

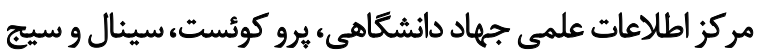

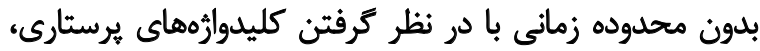

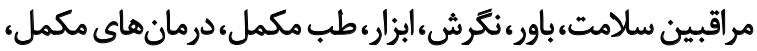

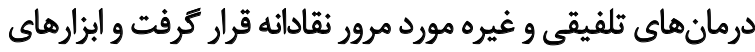

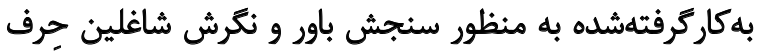

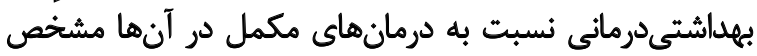

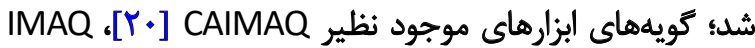

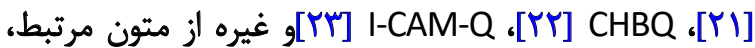

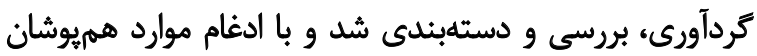

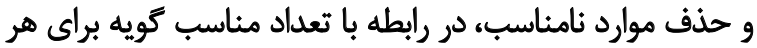

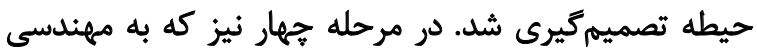

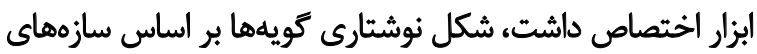

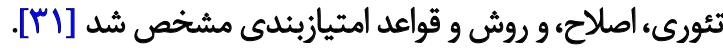

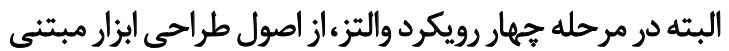

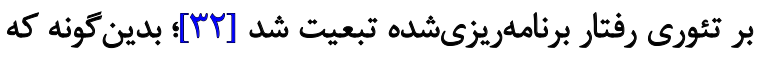

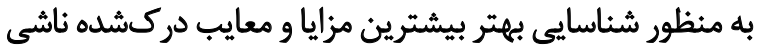

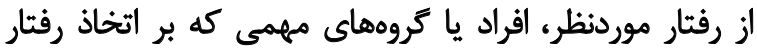

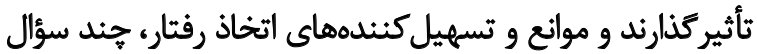

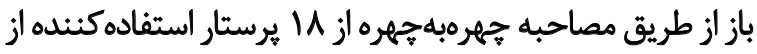

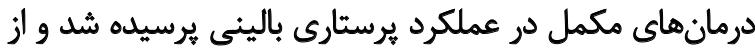

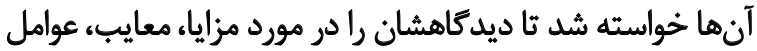

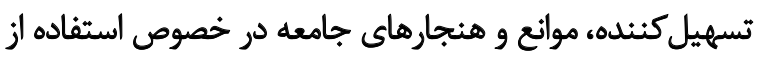

درمانهاى مكمل در مراقبت يرستارى از بيماران بيان كنيند.

سيس با استفاده از نتايج بهدستآمده بر اساس سه سازه

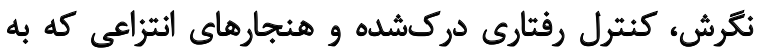

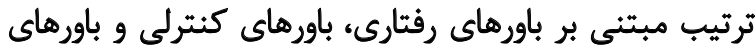

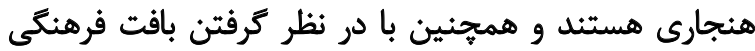

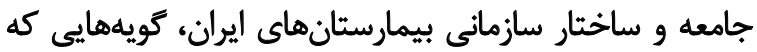

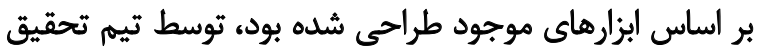

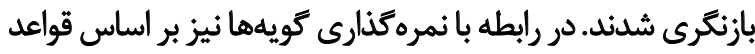

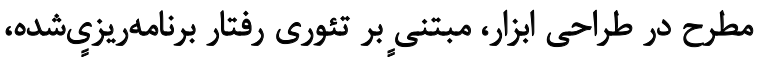

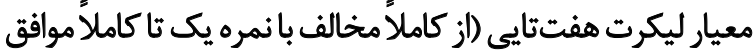

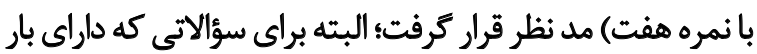
منفى بودند، نمرهدهى معكوس اعمال شدر.

$$
\text { فاز دوم: روان سئجى ابئار }
$$

اين فاز در قالب سه مرحله اجرا شد كه به شرح زير است.
از موضوعات مطرح، فرايند روانسنجى مناسبى دنبال نشده است

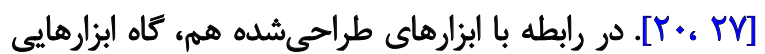

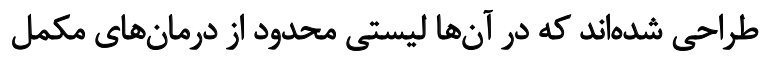

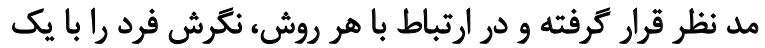

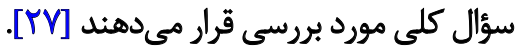

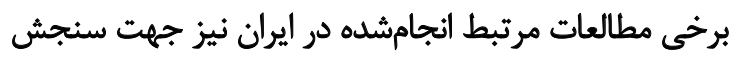

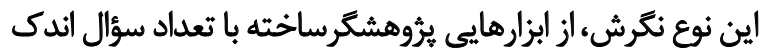

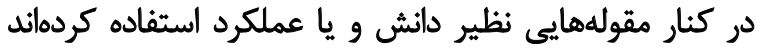

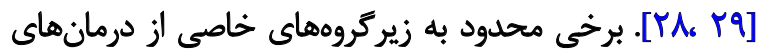

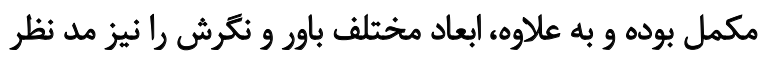

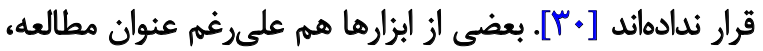

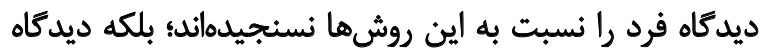

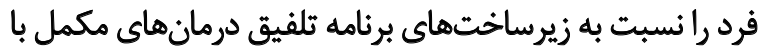

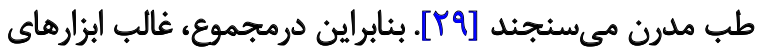

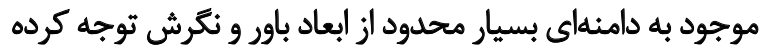

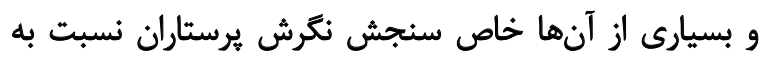
درمانهاى مكمل طراحى نشدهاند.

لحاظ كردن جارجوب نظرى مناسب در طراحى ابزارهاى

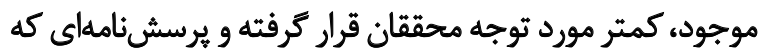

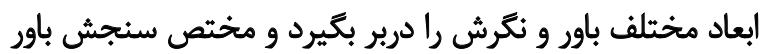

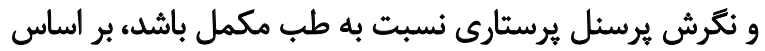

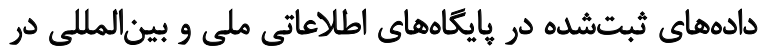

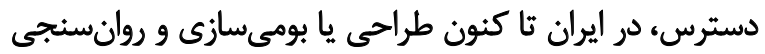

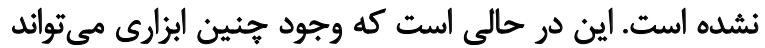

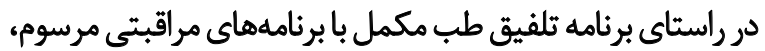

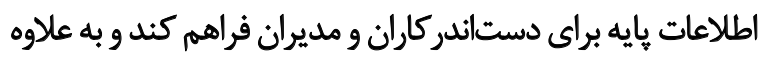

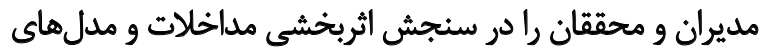

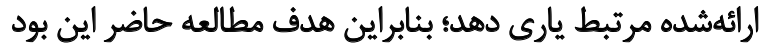

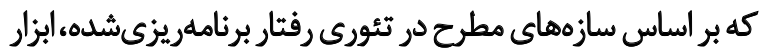

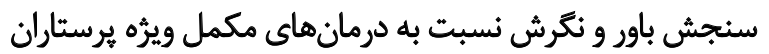
شاغل در عرصههاى بالينى ايران، طراحى و روانسنجى شئ شود.

\section{مواد و روشها}

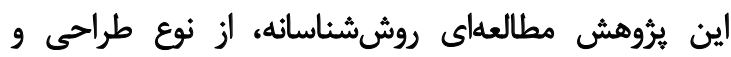
روانسنجى ابزرار بود كه در دو فاز به شرح زير اجرا شد. أند.

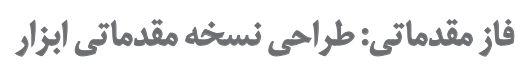

در اين فاز، اقدام به طراحى ابزار بر اساس رويكرد جهارمرحلهاى

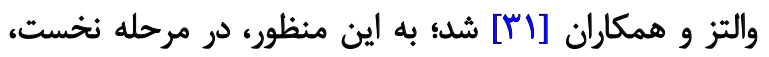

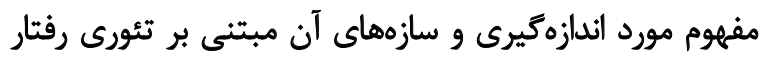

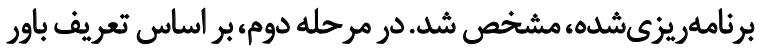

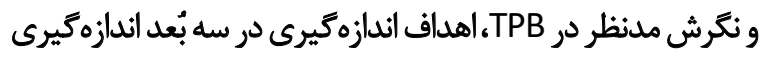
باورهاى كنترلى، باورهاى رفتارى و باورهاى هنجارى تعيين و در در 
مرحله Y: بررسى روايى سازه (تحليل عاملى و مقايسه كروههاى شناختهشده) و اثر سقف و كفي" سرازي"

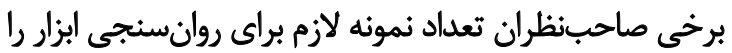

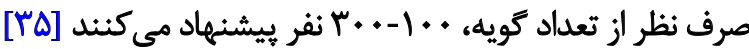

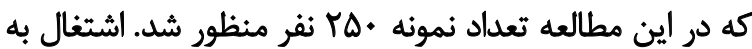

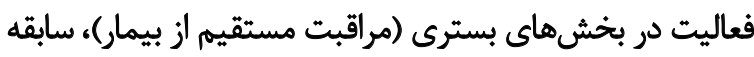

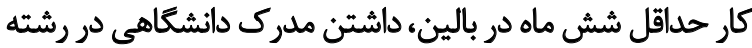

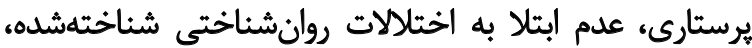

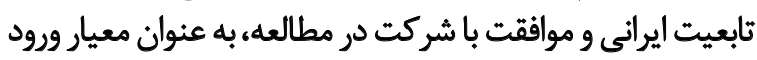

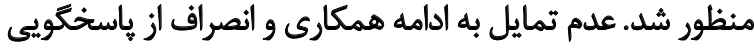
به يرسشنامه در حين تكميل آنه به عنوان معيار خروج در نظر اندراف كرفته شد.

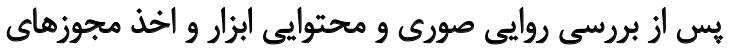

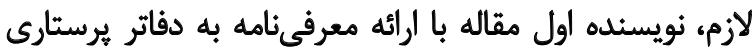

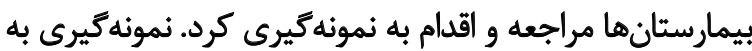

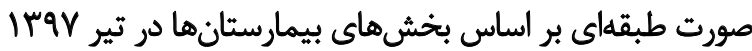

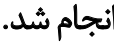

بخشهاى بالينى مختلف در بيمارستانهاى تحت يوشش

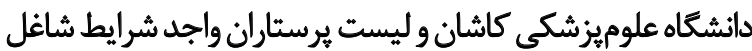

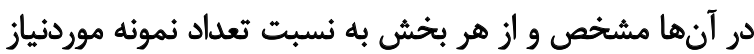

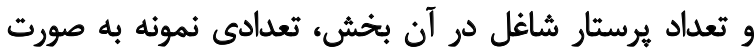

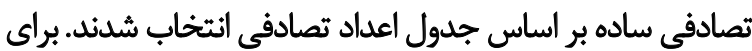

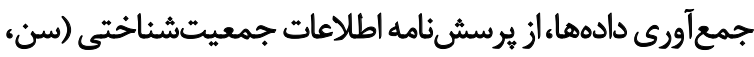

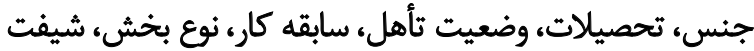

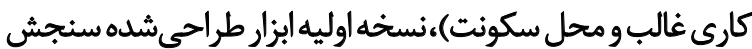

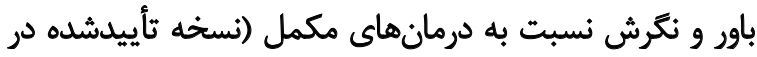

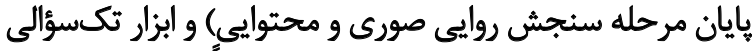

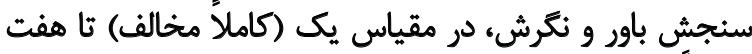

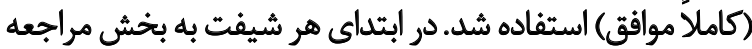

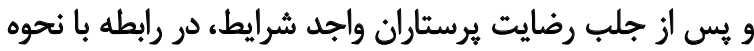

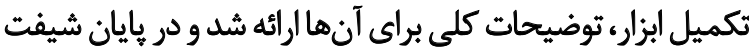

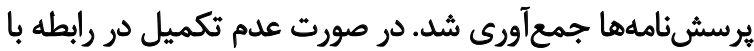

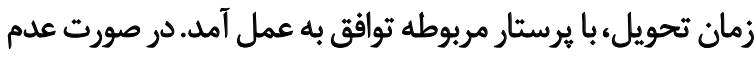

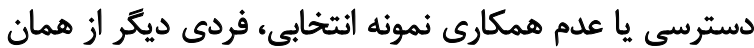
بخش به صورت تصادفى جايكزين شد.

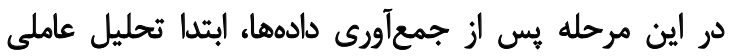

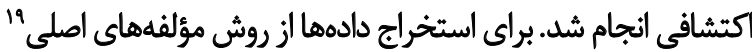
با قرخش واريماكس •r استفاده شد. به منظور تعيين تعداد عوامل،

18. Ceiling and Floor Effect

19. Principal Components

20. Varimax Rotation
مرحله ا: بررسى روايى صورى و محتوايیى

به منظور بررسى روايي محتوايى كيفى و كميى، نسخه

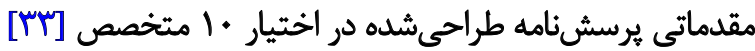

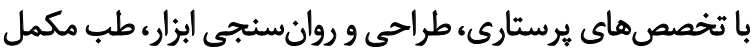

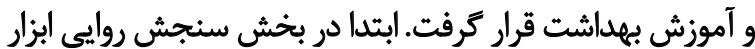

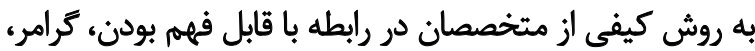

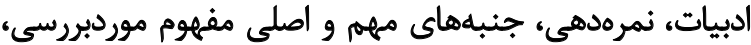

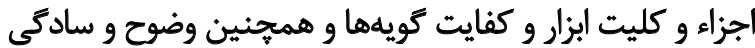
آنها نظرخواهى شد. بر اساس نظرات آنرات جمعآ آورى شده، تغييرات

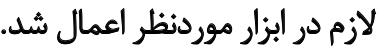

براى بررسى روايى محتوايى به روش كمى، از نسبت روايى إيى

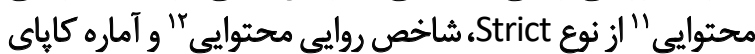

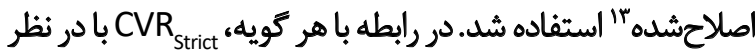

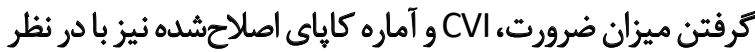

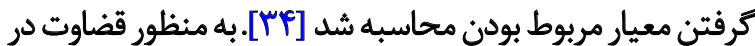

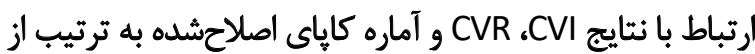

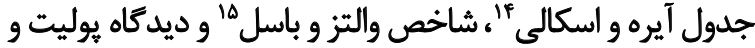

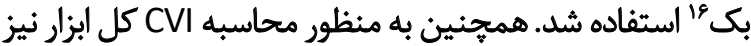
S-CVI Average

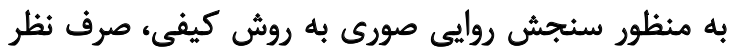

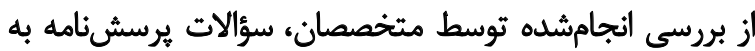

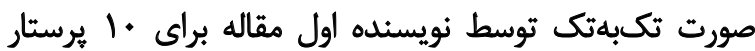

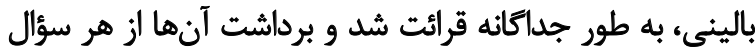

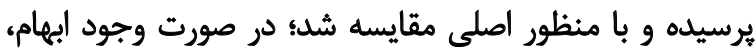

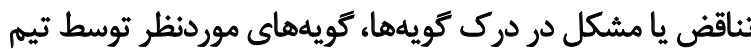

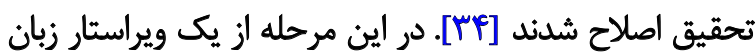

$$
\text { فارسى نيز كمك كرفته شد. }
$$

جهت سنجش روايى صورى كتّى نظرات بيرستاران مذكور

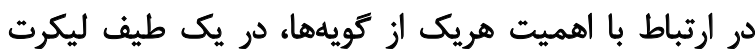

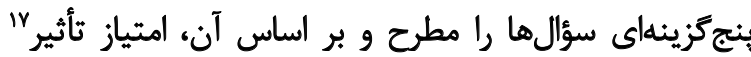

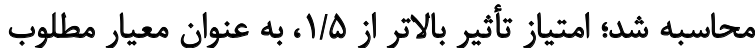

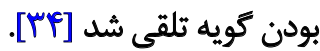

11. Content Validity Ratio (CVR)

12. Content Validity Index (CVI)

13. Modified Kappa Statistic

14. Ayre \& Scally (2014)

15. Waltz \& Bausell (1981)

16. Polit \& Beck (2012)

17. Item Impact 


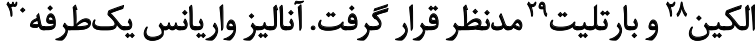

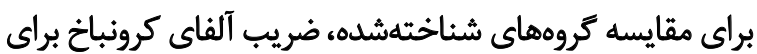

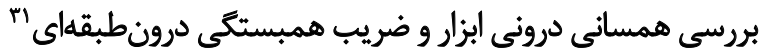

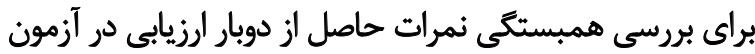

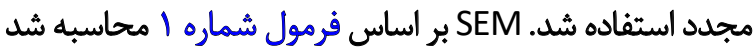

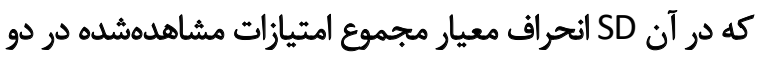

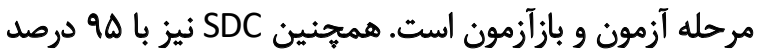

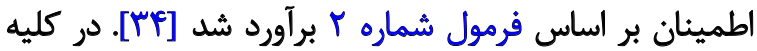

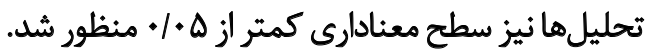

$S E M=S D \times V(1-I C C)$

$$
: r
$$

$S D C=1.96 \times \mathrm{V}(2 \times S E M)$

\section{يافتهها

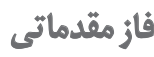

بيشنويس ابزار، داراى • ع كويه در سه حيطه باورهاى رفتارى،

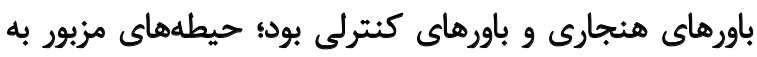

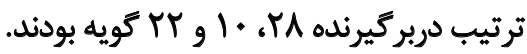

فأزوان سنجمي

\section{مرحله اول: بررسى رواييى صورى و محتوايى}

در بخش سنجش روايى محتوايى كيفى، شكل ظاهرى برخى

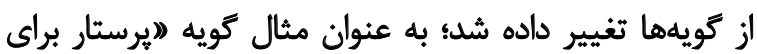

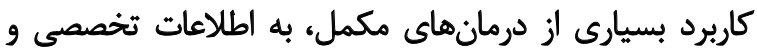

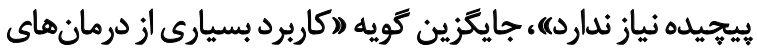

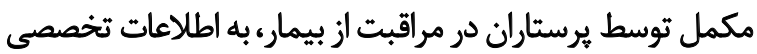

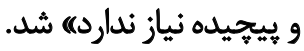

در بخش سنجش روايى محتوايى كمّى،

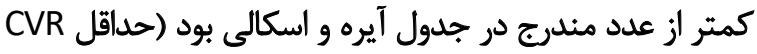

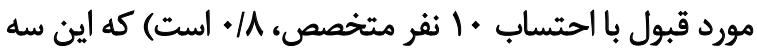

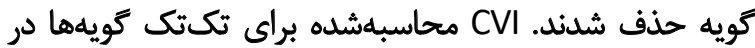

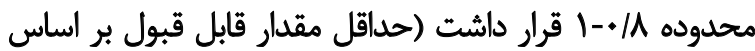

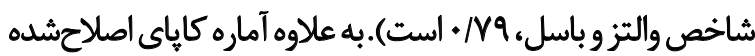

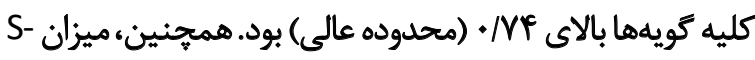
CVI Average

28. Kaiser-Meyer-Olkin (KMO)

29. Bartlett

30. Analysis of Variance (ANOVA)

31. Intraclass Correlation Coefficient (ICC)

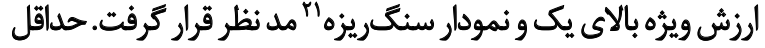

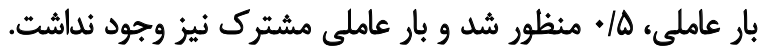

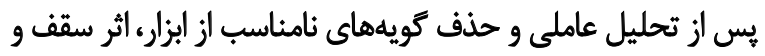

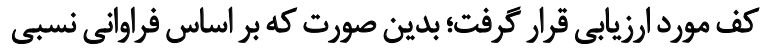

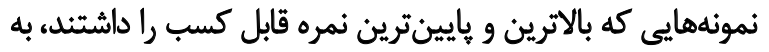

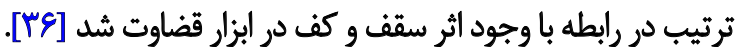
روش مقايسه گروههاى شناختهشده نيز براى بررسى روايى برائ

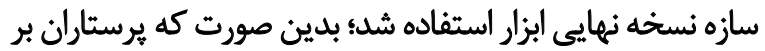

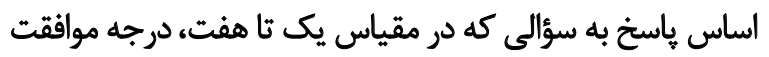

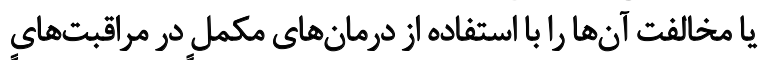

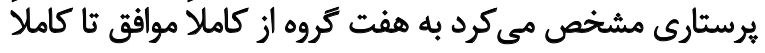

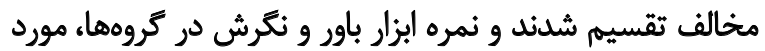
مقايسه قرار ترفت.

\section{مرحله بّ:سنجش بايايى (همسانى درونى و ثبات)}

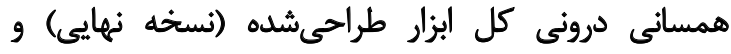

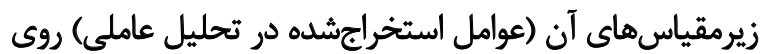

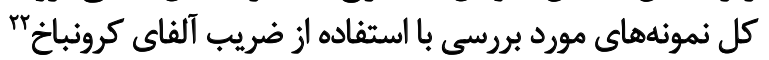
محاسبه شد.

براى سنجش ثبات ابزار، روش آزمون مجدد" بر به كار كرفته

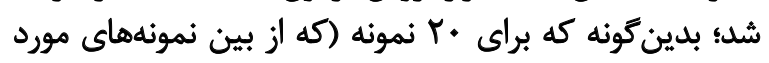

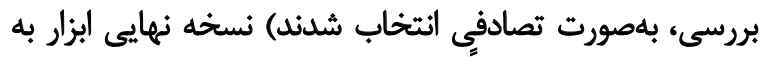

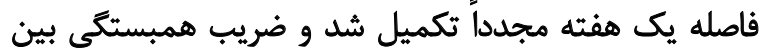

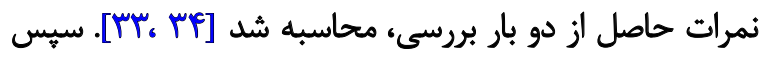

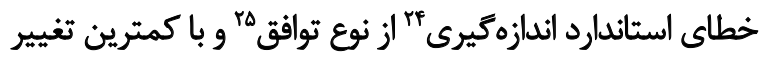

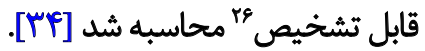

\section{تجيزيه و ثتحليل دادهها}

تجزيه و تحليل با استفاده از نسخه 19 نرمافزار

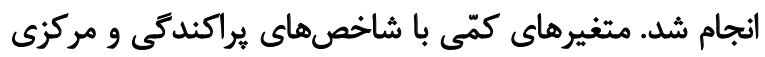

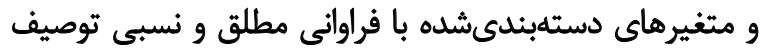

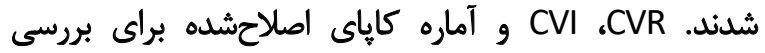

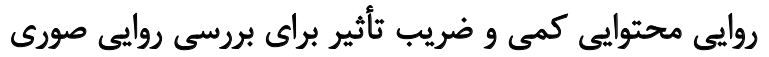

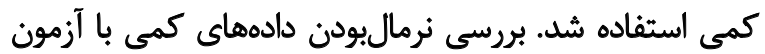

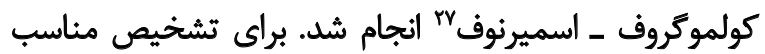

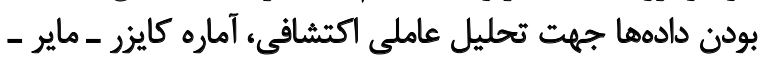

\section{Scree Plot}

22. Cronbach's Alpha

23. Test-retest

24. Standard Error of Measurement (SEM)

25. Agreement

26. Smallest Detectable Change (SDC)

27. Kolmogorov-Smirnov 


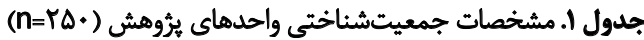

\begin{tabular}{|c|c|c|}
\hline تعداد (درصد) & \multicolumn{2}{|c|}{ متغير } \\
\hline$\Delta)(T \cdot / T)$ & مذكر & \multirow[b]{2}{*}{ جنس - } \\
\hline $199(19 / 8)$ & مؤنث & \\
\hline $19 \%(W / Y)$ & مثاهل & \multirow{4}{*}{ وضعيت تأهل } \\
\hline$\Delta \rho(K M / F)$ & مجرد & \\
\hline$\cdot(\cdot)$ & مطلقهه & \\
\hline $\left.1(\cdot /)^{*}\right)$ & 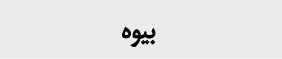 & \\
\hline$M(q M / Y)$ & كارشناسي & \multirow{3}{*}{ تحصيلات } \\
\hline $18(8 / 4)$ & كارشناسى ارشد & \\
\hline $1(\cdot / 9)$ & دكترى & \\
\hline$M(1 \% / \varepsilon)$ & اورزانس & \multirow{12}{*}{ بخش محل اشتغال } \\
\hline $\mathbb{P}(\mathrm{NA})$ & داخلى & \\
\hline 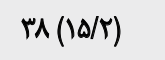 & جراحى عمومى & \\
\hline$\Leftrightarrow \&(T \& / K)$ & بخش هاى ويثره & \\
\hline$\Lambda(T / T)$ & 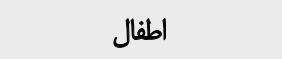 & \\
\hline$M(V / T)$ & 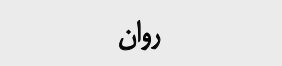 & \\
\hline$r(\cdot / A)$ & زنان و زايمان & \\
\hline $8(T / F)$ & عفوتى ع ع & \\
\hline$r(\cdot / \lambda)$ & انكولوزى & \\
\hline If $(\Delta / 9)$ & اتاق عمل & \\
\hline$f(1 / 8)$ & 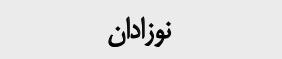 & \\
\hline $11(\varphi / \varphi)$ & جشه و كوش و حلق و يينى & \\
\hline$r f(q / 8)$ & كمتر از يك سال & \multirow{4}{*}{ سابقه كار بالينى } \\
\hline eI $(\pi / F)$ & يك ثنا ه سال & \\
\hline$F(M N)$ & هـا +ا سال & \\
\hline $119(F V / 9)$ & •ال سال و بالاتر & \\
\hline Mer (qV/T) & شهر & \multirow{2}{*}{ محل سكونت } \\
\hline$V(T / A)$ & | ل روستا & \\
\hline$M(T \Delta / r)$ & صبح & \multirow{3}{*}{ شيفت كارى غالب } \\
\hline$N(m e / 5)$ & عصر & \\
\hline$V \in(r \cdot / f)$ & شب & \\
\hline
\end{tabular}

|

بهتر بيمار با بيمارى كمك مي كند) شد. در بخش تعيين روايي

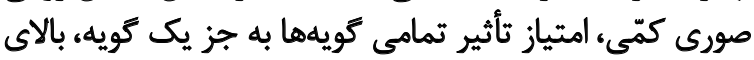

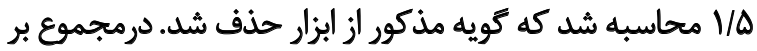

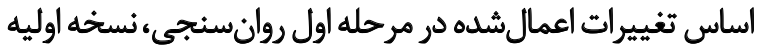
ابزار، ولاكويهاى تعيين شد. تفيرات

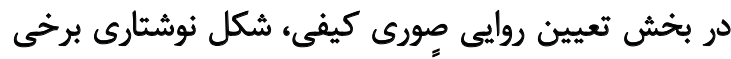

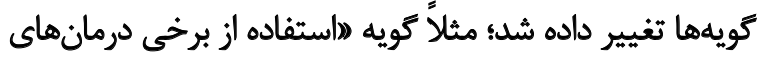

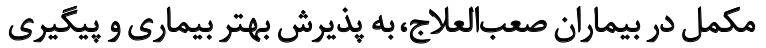

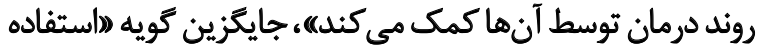
از برخى درمانهاي مكمل در بيماران صعبالعلاج، به ساز تارى 
جدول r. ارزش ويزهه و ميزان واريانس تبيينشده توسط عوامل استخراجشده از نسخه نهايى ابزار سئجش باور و نكرش برستاران نسبت به درمانهاي مكمل

\begin{tabular}{|c|c|c|}
\hline واريانس تيبينشده توسط هر عامل & ارزش ويزوه & عامل ل \\
\hline WFV & $1 . / m$ & عامل اول (وا سؤال) \\
\hline$V / \Delta A$ & $F / M$ & عامل دوم (و سوال) \\
\hline Vh. & $r / 9 V$ & عامل سوم (و سؤال) \\
\hline$s M^{e}$ & P/VA & عامل جهارم (" سووال) \\
\hline slor & $r / M r$ & عامل ئجمم (Vؤال) \\
\hline$\Delta / M^{m}$ & $r / M$ & عامل ششمى (ب سؤال) \\
\hline$\Delta T / T A$ & & كل عوامل \\
\hline
\end{tabular}

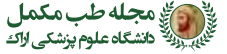

ابزار شناسايى كرد.اين عوامل تحت عناوين (إيامدهاى مربوط به

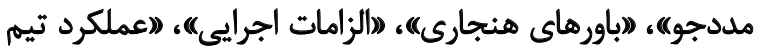

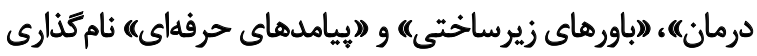

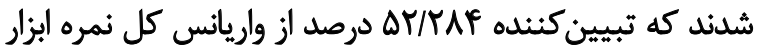

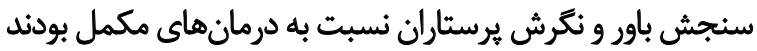

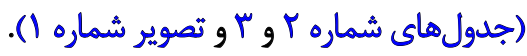

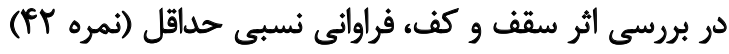

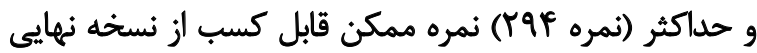

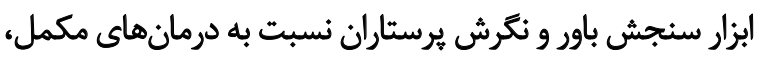

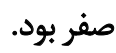

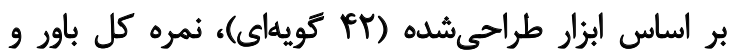

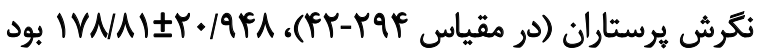

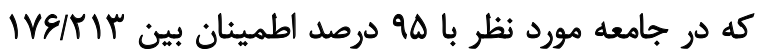

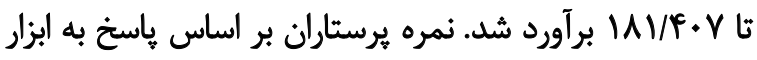

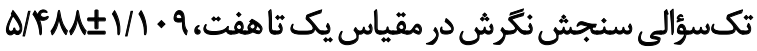

مرحله دوم: بررسى روايى سازه (تحليل عاملى و مقايسه كروهاي شناختهشده) و اثر سقف و كف درائ

دمر طول مدت نمونه

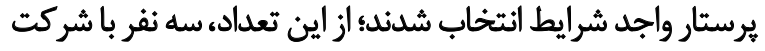

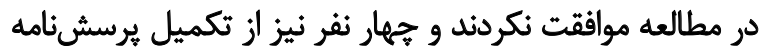

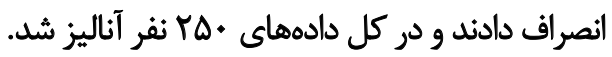

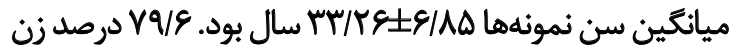

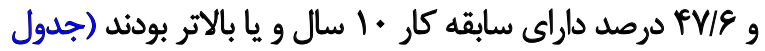

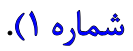
در رابطه با بررسى قابليت ابزار (نسخه عهامويهاى) براى تحليل

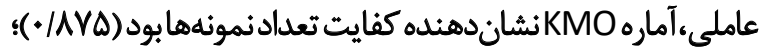

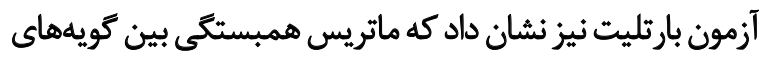

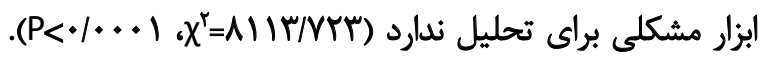

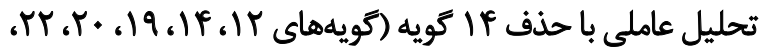
海

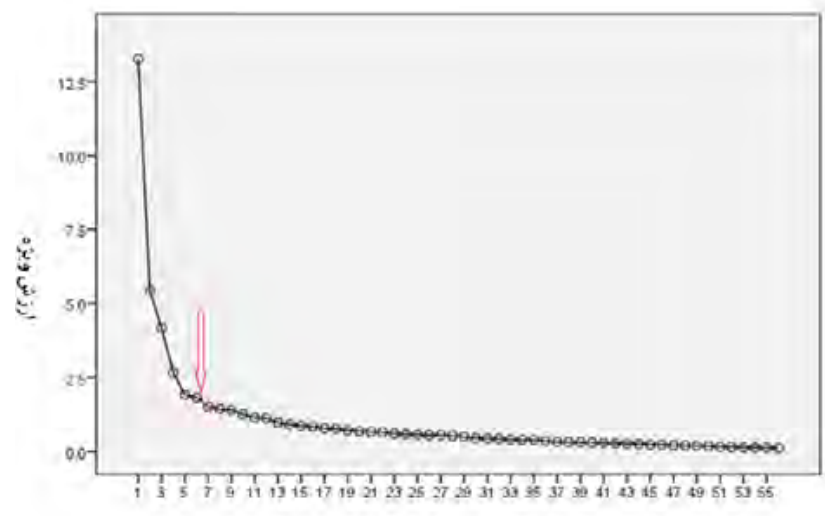

شعازء عامل 
جدول ب. كويهماي عوامل استخراجشده در تحليل عاملى ابزار سنجش باور و نكرش يرستاران نسبت به درمانهاي مكمل به همراه بار عاملى

\begin{tabular}{|c|c|c|c|c|c|c|c|}
\hline \multicolumn{6}{|c|}{ شماره عامل استخراجشده } & \multirow{2}{*}{\multicolumn{2}{|c|}{ كويه }} \\
\hline 7 & • & $\varepsilon$ & $r$ & $r$ & 1 & & \\
\hline & & & & & $\cdot M^{n}$ & 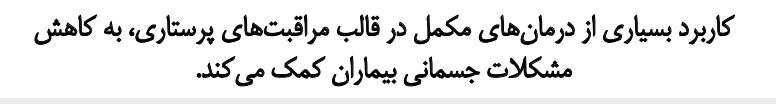 & 1 \\
\hline & & & & & $\cdot M$ & 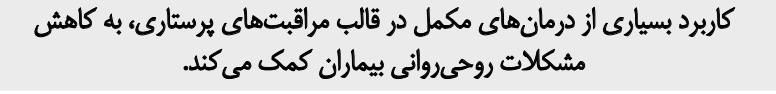 & $r$ \\
\hline & & & & & $\cdot M$ & اسثفاده از برخى درمانهاي مكمل براى بيماران، به القاى حس خوبيودن در آنها & $r$ \\
\hline & & & & &.$/ \Delta \mu^{\mu}$ & استفاده از برخى درمان هاى مكمل براى بيماران در حال احتضار، بله مرى آرام & r \\
\hline & & & & & $\cdot M$ & استفاده از برخى درمان هاى مكمل در بيماران، انتكيزه بهبود را أززايش مىدهد. & $\Delta$ \\
\hline & & & & & $\cdot / r$ & 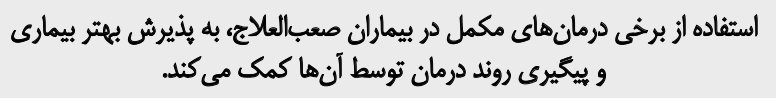 & 8 \\
\hline & & & & &.$M$ & 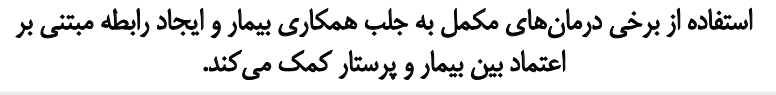 & $\checkmark$ \\
\hline & & & & & $\cdot / \mathrm{NA}$ & الستفاده از برخى درمانهاى مكمل در مددجو (بيمار و يا خانواده وى) ايجاد آرامش & $\wedge$ \\
\hline & & & & & $.18 \mathrm{~V}$ & استفاده از برخى درمانهاى مكمل در قالب مراقبتهاى يرستارى از نظر علمى & 9 \\
\hline & & & & & .189 & 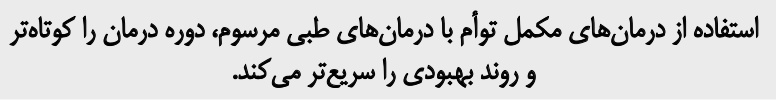 & 1. \\
\hline & & & & & .180 & استفاده از درمان هاى مكمل، طول مدت بسترى در بيمارستان را كاهش ميدهد. & 11 \\
\hline & & & & & $\cdot M$ & برخى درمانهاي مكمل در ييشكيرى أز بعضى هشكلات جسمائى در بيماران هؤثر & ir \\
\hline & & & & & $\cdot \mathrm{MA}$ & برخى درمانهاي مكمل در ييشكيرى از بعضى مشكلات روحيروائى در بيماران & Ir \\
\hline & & & & & & اكر مشكلات بيمار با درمان هاى طبى مرسوم بهتر نشود، يرستاران بايد درمان هاى إمتحان & if \\
\hline & & & & & .108 & كاربرد بسيارى از درمانهاى مكمل در قالب مراقبتهاى يرستارى براى ملدجويان & 10 \\
\hline & & & & & .10 & اثربخشى بسيارى از درمانهاي مكمل در مقايسه با طب مرسوم بيشتر است. & 18 \\
\hline . $\mathrm{HN}$ & & & & & & استفاده از درمانهاي مكمل در عملكرد بالينى به اسثقالال حرقهاى يرستار كمى & IV \\
\hline$\cdot / \Delta F$ & & & & & & اسثفاده از درمانهاى مكمل در عملكرد بالينى به معرفي بيرستار به عنوان يك فرد & IA \\
\hline & & & & & & درمان هاى مكمل، تهديدكننده سلامت عمومى در جامعه هستند. & 19 \\
\hline
\end{tabular}




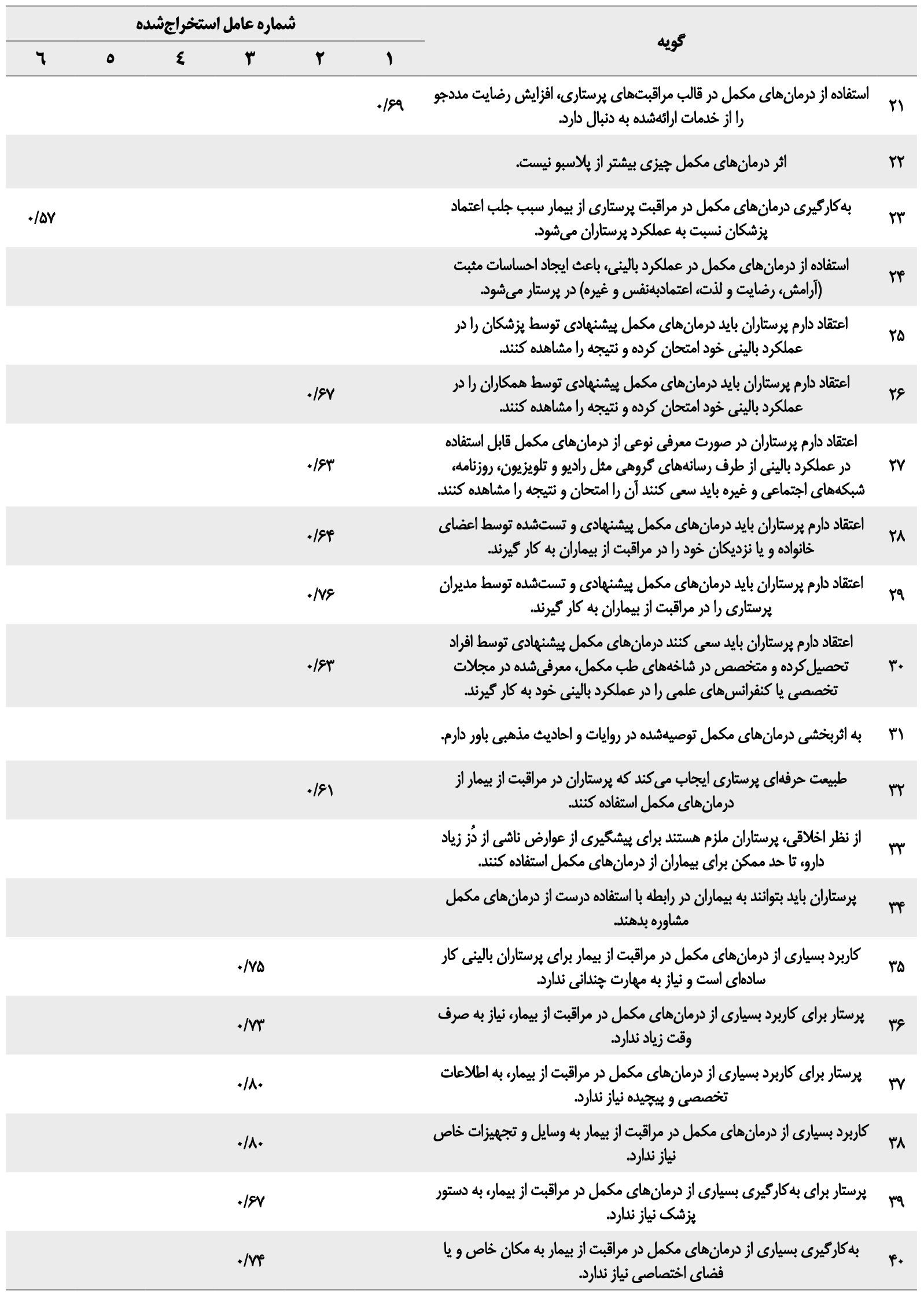




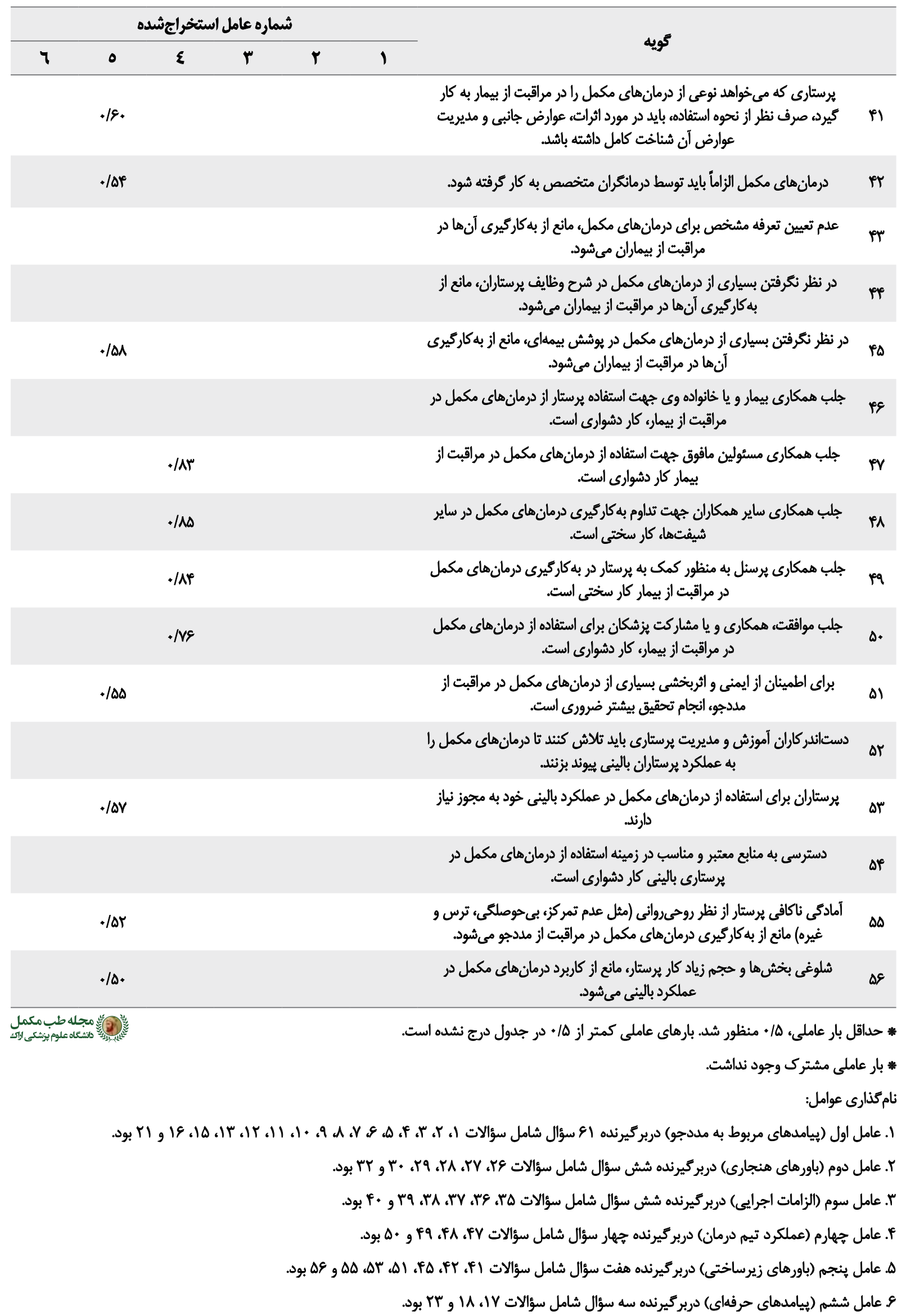




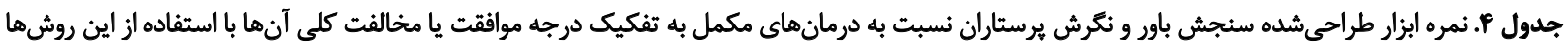

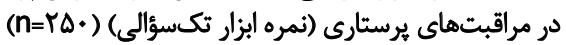

\begin{tabular}{|c|c|c|c|c|c|c|c|c|}
\hline \multirow{4}{*}{ واريجيه أزمون أناليز } & \multicolumn{7}{|c|}{ 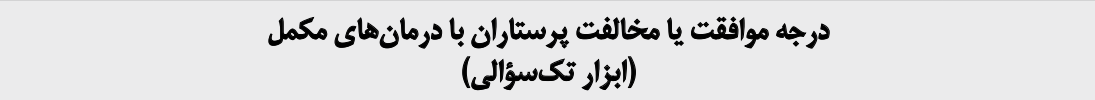 } & \multirow{4}{*}{ 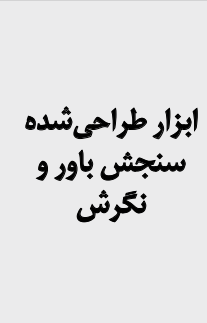 } \\
\hline & $v$ & 7 & - & $\varepsilon$ & $r$ & $r$ & 1 & \\
\hline & كاملاً موافق & موافق & تا مدودى موافق & نظرى نداريم & تا مدودى مخالف & مخالف & كاملاً مخالف & \\
\hline & $(n=\varepsilon \cdot)$ & $(n=q 1)$ & $\left(\mathrm{n}=\mathrm{V}_{0}\right)$ & $(n=r \varepsilon)$ & $(n=\Lambda)$ & $(n=\varepsilon)$ & $(\mathrm{n}=1)$ & \\
\hline $\begin{array}{c}F=\& / 1 \Delta \\
P<+1+\cdots+1 *\end{array}$ & $\begin{array}{l} \pm r \Delta / q . \\
M Y Y / \Lambda Q\end{array}$ & $\begin{array}{l} \pm \text { IV/9 } \\
\text { Wr/g8 }\end{array}$ & $\begin{array}{l} \pm \mid q / 4 \\
|R / \Delta|\end{array}$ & $\begin{array}{l} \pm \text { if/\&A } \\
\text { inf/gr. }\end{array}$ & $\begin{array}{l} \pm r \cdot / M V \\
1 \Delta \& / \& r\end{array}$ & $\begin{array}{l} \pm r q / T \Delta \\
1 \& r / \Delta\end{array}$ & ind & 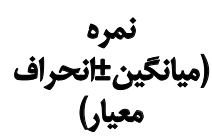 \\
\hline
\end{tabular}

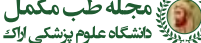

* جهت انجام تست تعقيبى، كروه يك به علت داشتن يك نمونه حذف شد.

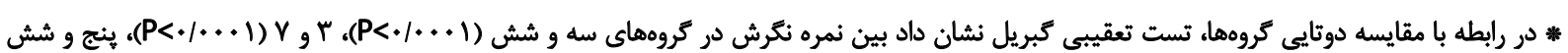

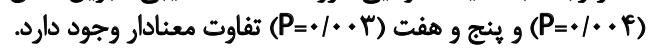

نمره باور و نكرش را در مقياس YF تأ FqF اندازهكيرى كند. بيشنويس ابزار سنجش باور و نكرش برستاران نسبت به به بهان

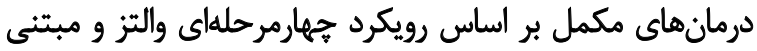

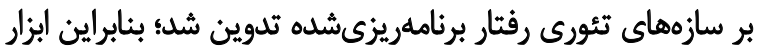

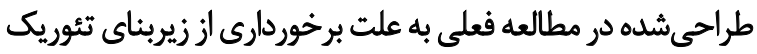

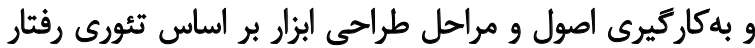

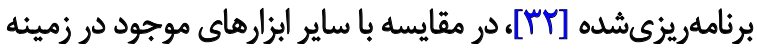

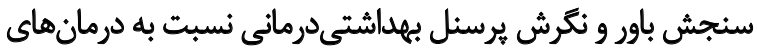

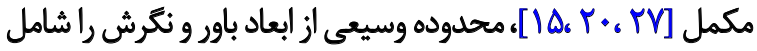

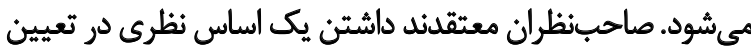

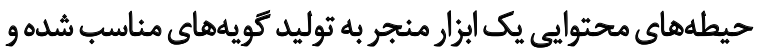

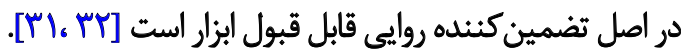
در بخش سنجش روايى محتوايى، با بهرهيرى از نظرات

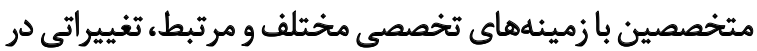

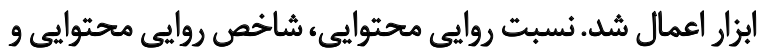

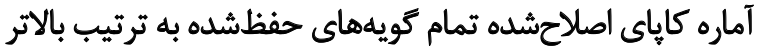

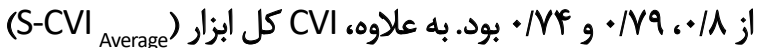

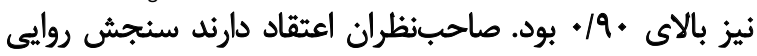

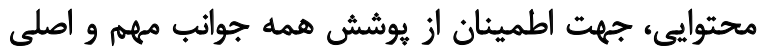

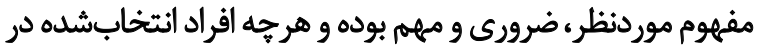

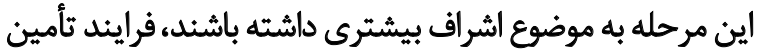

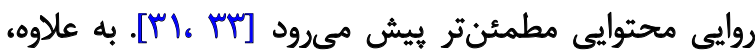

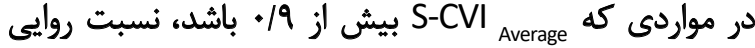

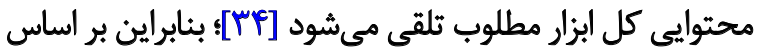

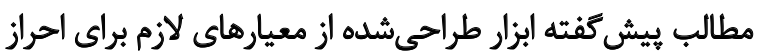
روايى محتوايى برخوردار است.

در بخش سنجش روايي صورى بر اساس مسائل مطرحشده

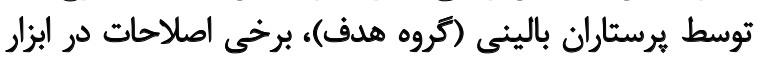

محاسبه شد؛ بر اساس آزمون اسيّيرمن - براون بَ بين نمره ابزار

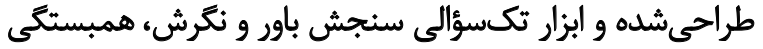

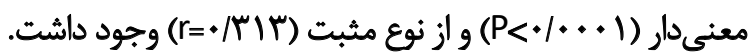
در روش مقايسه كروههاى شناختهشده، آناليز واريانس يكسطرفه

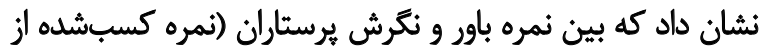

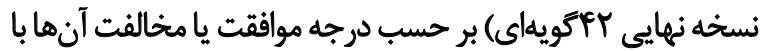

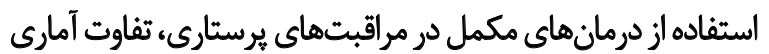

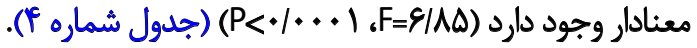

\section{مرحله بّ: هممانى درونى و ثبات}

در بخش سنجش همسانى درونى، ضريب آلفاى كرونباخ كل

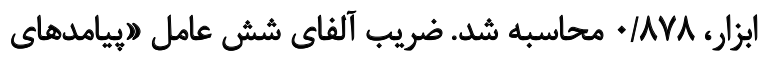

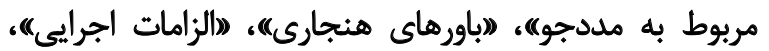

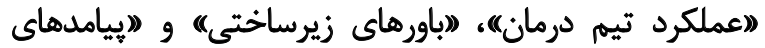

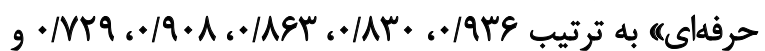

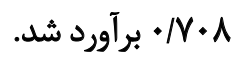

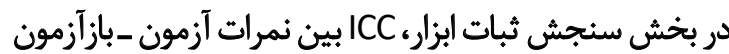

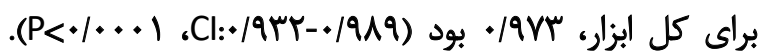

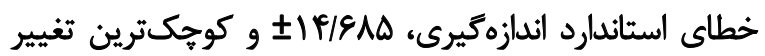
واقعى قابل تشخيص نيز با هو درصد اطمينان، بع/. أمحاسبه شُّد.

\section{$\leftrightarrow$}

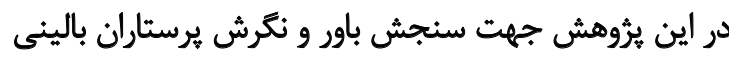

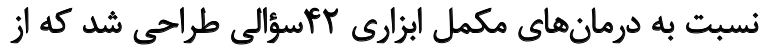

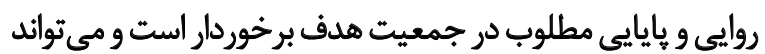

32. Spearman-Brown 
كه تفاوت معنى دار مشاهده شود، روايى ساختارى ابزار مورد تأييد

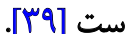

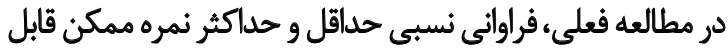

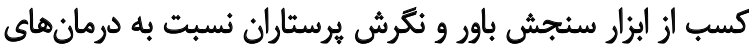

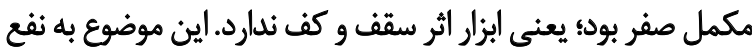

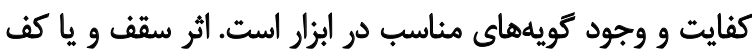

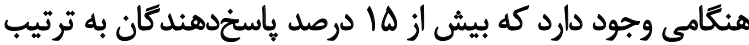

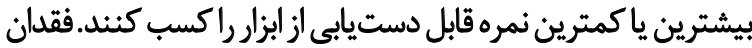

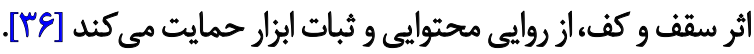
در مطالعه ماضريب آلفاي كرونباخ كل ابزار لهVA • برآورد شد.

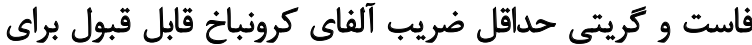

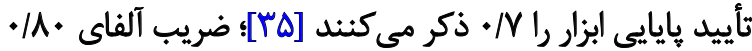

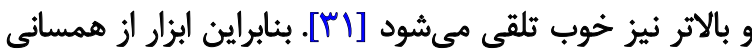
درونى مطلوبى برخوردار است.

ضريب همبستكى بين نمرات حاصل از دو نوبت آزمون

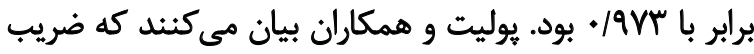

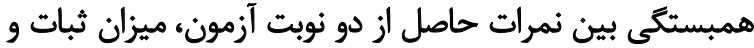

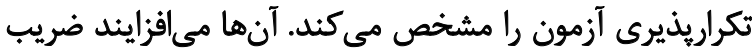

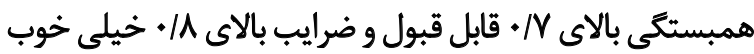

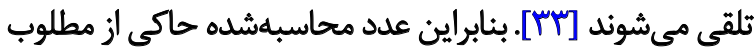

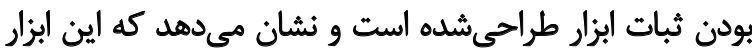

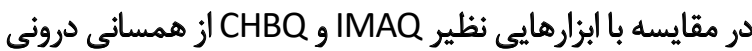

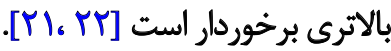

در مطالعه حاضر، خطاى استاندارد اندازميرى و كمترين تغيير

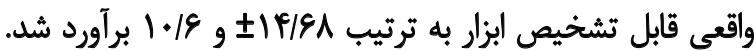

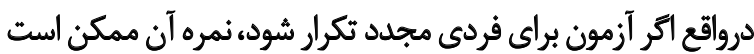

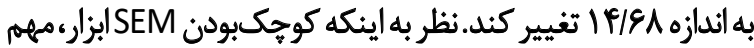

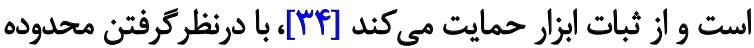

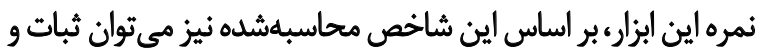
تكراريذيرى و در اصل يايايى آن را مطلوب ثلقيى كرد.

استفاده از تئورى رفتار برنامهريزىشده در تعريف مفهوم باور

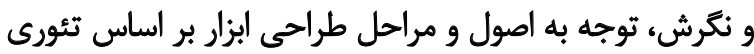

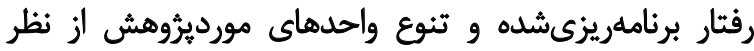

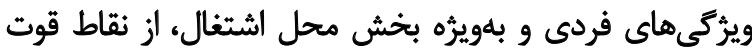

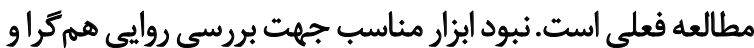

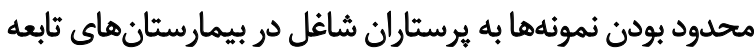

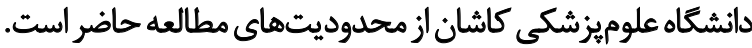

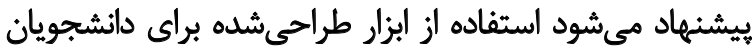

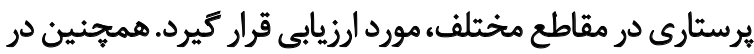

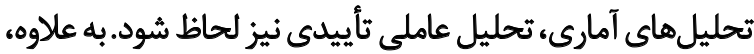

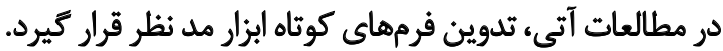

انجام شد. همجنين امتياز تأثير كليه كويههاي حفظشده در ابزار

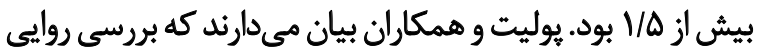

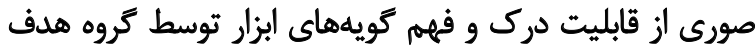

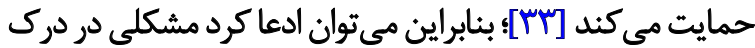
كويههاى ابزار طراحى

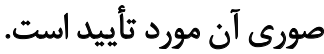

تحليل عاملى اكتشافى، وجود شش سازه هائيامدهاى مربوط به

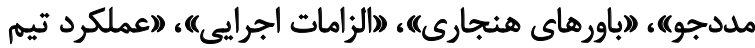

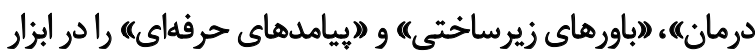

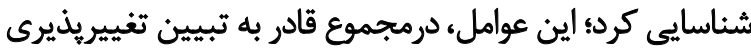

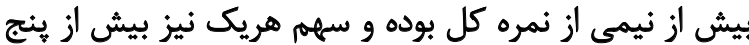

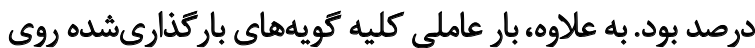
عوامل نيز بالاى ه/ • بود و بار عاملى مشترك هم وجليه كويهاى باركنارد نداشت. برخى صاحبنظران بيان مى كنند كه اكر عوامل شناسايىشده

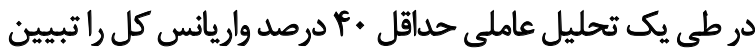

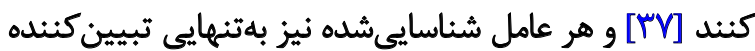

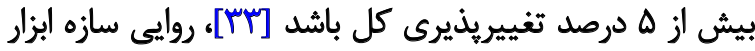

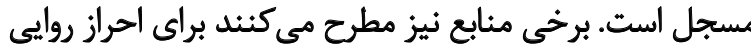

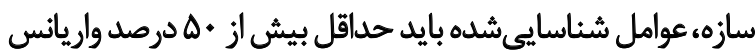

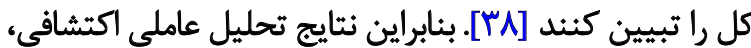

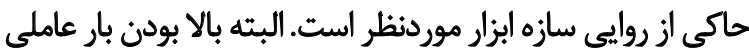

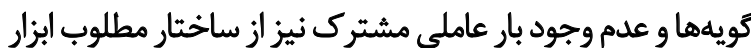

حمايت مى كند [TH]

محتواى كويههاى باركذارىشده روى عوامل استخراجشده

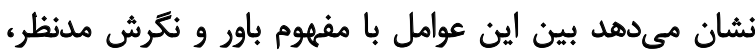

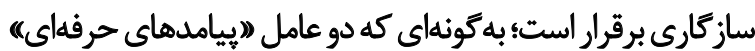

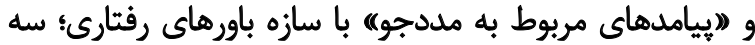

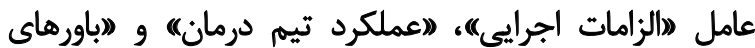

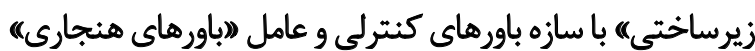

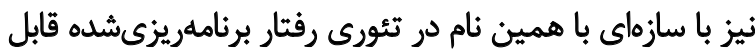

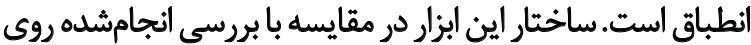

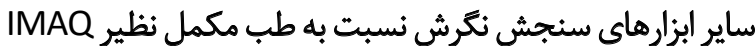

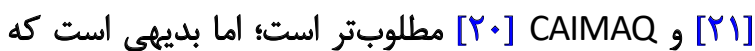

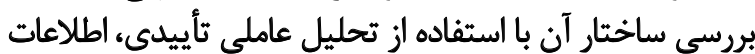
بهتر و دقيقترى را فراهم مى كند.

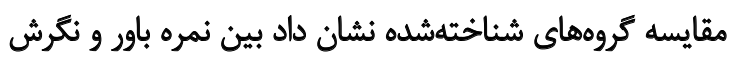

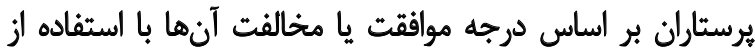

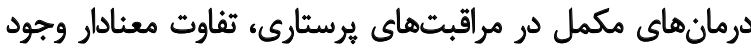

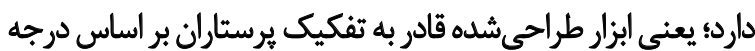

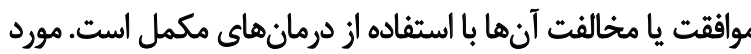

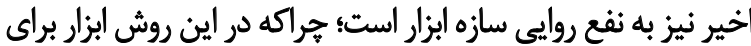

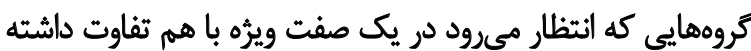

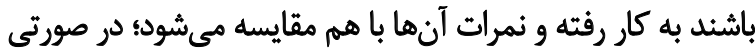




\section{نتيجلتيرى}

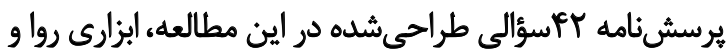

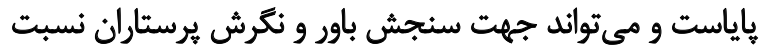
به درمانهاي مكمل استفاده شود.

\section{مالاحظات اخلاقى}

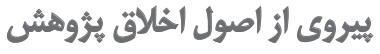

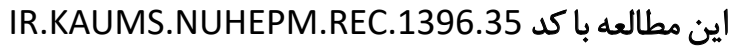
در كميته اخلاق آن دانشكاه تصويب شد. به تمام واحدهاي النداي

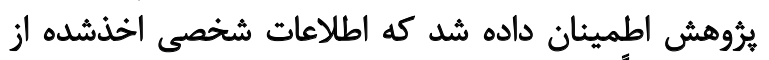

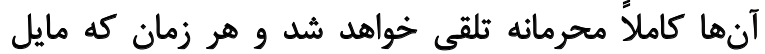

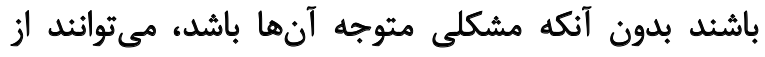

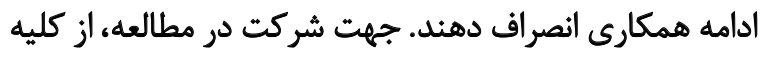
نمونهها رضايت آكاهانه كتبى كرفته شداف

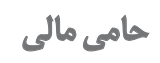

مطالعه حاضر بخشى از ياياننامه نويسنده اول مقاله در رشته

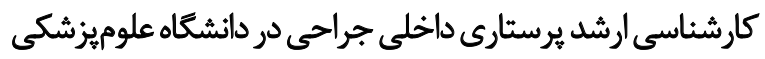

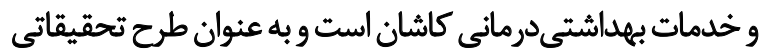

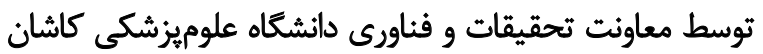
مورد حمايت مالى قرار ترفته است (شمار تواره طرح:

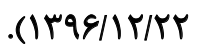

$$
\text { مشاركت ثويسند انَّان }
$$

طراحى مطالعه، جمعآورى دادهها و نكارش بيشيشنويس مقاله:

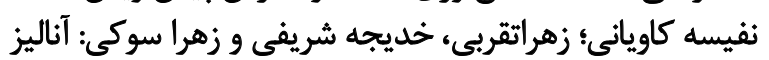

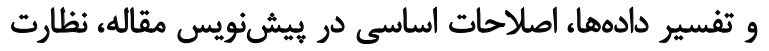

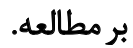

$$
\text { ت تعارض مناقع }
$$

در اين يُروهش هيجّونه تضاد منافعى وجود ندارد.

$$
\text { تشكر و قدردانى }
$$

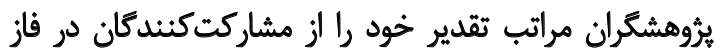

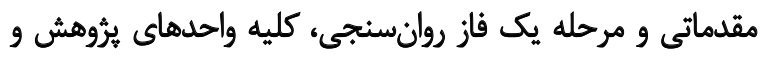

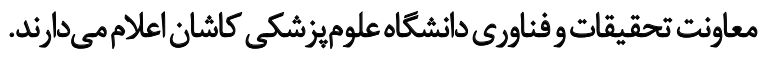




\section{References}

[1] Ben-Arye E, Cassileth B, Heusser P, Afifi F, Saad B, Selvan SR. Complementary and integrative oncology in the cross-cultural region of the Middle East and South Asia. Evidence-Based Complementary and Alternative Medicine. 2012; 2012:940961. [DOI:10.1155/2012/940961] [PMID] [PMCID]

[2] Steel A, McIntyre E, Harnett J, Foley H, Adams J, Sibbritt D, et al. Complementary medicine use in the Australian population: Results of a nationally-representative cross-sectional survey. Scientific Reports. 2018; 8(1):17325. [DOI:10.1038/s41598-018-35508-y] [PMID] [PMCID]

[3] Lee JA, Sasaki Y, Arai I, Go HY, Park S, Yukawa K, et al. An assessment of the use of complementary and alternative medicine by Korean people using an adapted version of the standardized international questionnaire (I-CAM-QK): A cross-sectional study of an internet survey. BMC Complementary and Alternative Medicine. 2018; 18(1):238. [DOI:10.1186/s12906-018-2294-6] [PMID] [PMCID]

[4] Shumer G, Warber S, Motohara S, Yajima A, Plegue M, Bialko M, et al. Complementary and alternative medicine use by visitors to rural Japanese family medicine clinics: Results from the international complementary and alternative medicine survey. BMC Complementary and Alternative Medicine. 2014; 14:360. [DOI:10.1186/1472-6882-14-360] [PMID] [PMCID]

[5] Kemppainen LM, Kemppainen TT, Reippainen JA, Salmenniemi ST, Vuolanto PH. Use of complementary and alternative medicine in Europe: Health-related and sociodemographic determinants. Scandinavian Journal of Public Health. 2018; 46(4):448-55. [DOI:10.1177/1403494817733869] [PMID] [PMCID]

[6] Anbari K, Gholami M. Evaluation of trends in the use of complementary and alternative medicine in health centers in Khorramabad (West of Iran). Global Journal of Health Science. 2016; 8(2):72-6. [DOI:10.5539/ gjhs.v8n2p72] [PMID] [PMCID]

[7] Shorofi SA. Complementary and alternative medicine (CAM) among hospitalised patients: Reported use of CAM and reasons for use, CAM preferred during hospitalisation, and the socio-demographic determinants of CAM users. Complementary Therapies in Clinical Practice. 2011; 17(4):199-205. [DOI:10.1016/j.ctcp.2011.05.001] [PMID]

[8] Yalcin S, Hurmuz P, McQuinn L, Naing A. Prevalence of complementary medicine use in patients with cancer: A Turkish comprehensive cancer center experience. Journal of Global Oncology. 2018; 4:1-6. [DOI:10.1200/JGO.2016.008896] [PMID] [PMCID]

[9] Amirmoezi F, Araghizadeh M, Mohebbinia Z, Kamfiroozi R, Haghpanah $\mathrm{S}$, Bordbar MR. Use of complementary and alternative medicine among Iranian cancer patients in south of Iran. International Journal of Cancer Management. 2017; 10(10):e7233. [DOI:10.5812/ijcm.7233]

[10] Hashempur MH, Heydari M, Mosavat SH, Heydari ST, Shams M. Complementary and alternative medicine use in Iranian patients with diabetes mellitus. Journal of Integrative Medicine. 2015; 13(5):319-25. [DOI:10.1016/S2095-4964(15)60196-0]

[11] Christina J, Abigail W, Cuthbertson LA. Nurses' knowledge and attitudes toward complementary therapies for cancer: A review of the literature. Asia-Pacific Journal of Oncology Nursing. 2016; 3(3):241-51. [DOI:10.4103/2347-5625.189816] [PMID] [PMCID]

[12] Yom YH, Lee KE. A comparison of the knowledge of, experience with and attitudes towards complementary and alternative medicine between nurses and patients in Korea. Journal of Clinical Nursing. 2008; 17(19):2565-72. [DOI:10.1111/j.1365-2702.2007.02065.x]

[13] Chang HY, Chang HL. A review of nurses' knowledge, attitudes, and ability to communicate the risks and benefits of complementary and alternative medicine. Journal of Clinical Nursing. 2015; 24(11-12):1466 78. [DOI:10.1111/jocn.12790] [PMID]

[14] Salomonsen L, Skovgaard L, La Cour S, Nyborg L, Launs $\varnothing$ L, Fønneb V. Use of complementary and alternative medicine at Norwegian and Danish hospitals. BMC Complementary and Alternative Medicine. 2011; 11:4. [DOI:10.1186/1472-6882-11-4] [PMID] [PMCID]

[15] Wilson LAM, White KM, Hamilton K. Predicting psychologists' intentions to integrate complementary and alternative therapies into their practice. Australian Psychologist. 2013; 48(2):149-58. [DOI:10.1111/ j.1742-9544.2011.00058.x]

[16] Godin G, Beaulieu D, Touchette JS, Lambert LD, Dodin S. Intention to encourage complementary and alternative medicine among gen eral practitioners and medical students. Behavioral Medicine. 2007 33(2):67-79. [DOI:10.3200/BMED.33.2.67-79] [PMID]

[17] Kam LYK, Knott VE, Wilson C, Chambers SK. Using the theory of planned behavior to understand health professionals' attitudes and intentions to refer cancer patients for psychosocial support. Psycho-Oncology. 2012; 21(3):316-23. [DOI:10.1002/pon.1897] [PMID]

[18] Ajzen I. The theory of planned behaviour: Reactions and reflections. Psychology \& Health. 2011; 29(6):1113-27. [DOI:10.1080/08870446.20 11.613995] [PMID]

[19] Taylor D, Bury M, Campling N, Carter S, Garfied S, Newbould J, et al. A review of the use of the Health Belief Model (HBM), the Theory of Reasoned Action (TRA), the Theory of Planned Behaviour (TPB) and the Trans-Theoretical Model (TTM) to study and predict health related behaviour change. London: National Institute for Health and Clinical Excellence; 2006. https://www.researchgate.net/publication/334114235

[20] Soleimani R, Jalali MM, Ahmadi R. [Attitude of clinical medical students and residents of Guilan University of Medical Sciences about complementary and alternative medicine (Persian)]. Research in Medical Education. 2013; 5(1):13-21. [DOI:10.18869/acadpub.rme.5.1.13]

[21] Schneider CD, Meek PM, Bell IR. Development and validation of IMAQ: Integrative Medicine Attitude Questionnaire. BMC Medical Education. 2003; 3:5. [DOI:10.1186/1472-6920-3-5] [PMID] [PMCID]

[22] Lie D, Boker J. Development and validation of the CAM Health Belief Questionnaire (CHBQ) and CAM use and attitudes amongst medical students. BMC Medical Education. 2004; 4:2. [DOI:10.1186/1472-69204-2] [PMID] [PMCID]

[23] Quandt SA, Verhoef MJ, Arcury TA, Lewith GT, Steinsbekk A, Kristoffersen $A E$, et al. Development of an international questionnaire to measure use of complementary and alternative medicine (I-CAM-Q). Journal of Alternative and Complementary Medicine. 2009; 15(4):3319. [DOI:10.1089/acm.2008.0521] [PMID] [PMCID]

[24] Rojas-Cooley MT, Grant M. Complementary and alternative medicine: Oncology nurses' knowledge and attitudes. Oncology Nursing Forum. 2009; 36(2):217-24. [DOI:10.1188/09.ONF.217-224] [PMID]

[25] Quartey NK, Ma PHX, Chung VCH, Griffiths SM. Complementary and alternative medicine education for medical profession: Systematic review. Evidence-Based Complementary and Alternative Medicine. 2012; 2012:656812. [DOI:10.1155/2012/656812] [PMID]

[26] McFadden KL, Hernández TD, Ito TA. Attitudes toward complementary and alternative medicine influence its use. Explore (NY). 2010; 6(6):380 8. [DOI:10.1016/j.explore.2010.08.004] [PMID] [PMCID]

[27] Balouchi A, Rahnama M, Hastings-Tolsma M, Shoja MM, Bolaydehyi E. Knowledge, attitude and use of complementary and integrative health strategies: A preliminary survey of Iranian nurses. Journal of Integrative Medicine. 2016; 14(2):121-7. [DOI:10.1016/S2095-4964(16)60245-5] 
[28] Adib-Hajbaghery M, Hoseinian M. Knowledge, attitude and practice toward complementary and traditional medicine among Kashan health care staff, 2012. Complementary Therapies in Medicine. 2014; 22(1):126-32. [DOI:10.1016/j.ctim.2013.11.009] [PMID]

[29] Barikani A, Beheshti A, Javadi M, Yasi M. Knowledge, attitude and practice of general practitioners toward complementary and alternative medicine: A cross-sectional study. Acta Medica Iranica. 2015; 53(8):501-6. [PMID]

[30] Rabani Khorasgani S, Moghtadaie L. Investigating knowledge and attitude of nursing students towards Iranian traditional medicine-case study: Universities of Tehran in 2012-2013. Global Journal of Health Science. 2014; 6(6):168-77. [DOI:10.5539/gjhs.v6n6p168] [PMID] [PMCID]

[31] Waltz CF, Strickland OL, Lenz ER. Measurement in nursing and health research. New York: Springer Publishing Company; 2010. https://books. google.com/books?id=1xAdjkR14ocC\&dq

[32] Francis J, Eccles MP, Johnston M, Walker AE, Grimshaw JM, Foy R, et al. Constructing questionnaires based on the theory of planned behaviour: A manual for health services researchers. Newcastle upon Tyne, UK: Centre for Health Services Research, University of Newcastle upon Tyne; 2004. https://openaccess.city.ac.uk/id/eprint/1735/

[33] Polit DF, Beck CT. Nursing research: Generating and assessing evidence for nursing practice. Philadelphia: Lippincott Williams \& Wilkins; 2012. https://books.google.com/books?id=hFGaSQAACAAJ\&dq

[34] Ebadi A, Zarshenas L, Rakhshan M, Zareian A, Sharifnia SH, Mojahedi M. [Principles of scale development in health science (Persian)]. Tehran: Jame'e Negar; 2017. http://opac.nlai.ir/opacprod/bibliographic/4685542

[35] Fawcett J, Garity J. Evaluating research for evidence-based nursing practice. Philadelphia: F.A. Davis; 2009. https://books.google.com/ books?id=PulcOQAACAAJ\&dq

[36] Terwee CB, Bot SDM, de Boer MR, van der Windt DAWM, Knol DL, Dekker J, et al. Quality criteria were proposed for measurement properties of health status questionnaires. Journal of Clinical Epidemiology. 2007; 60(1):34-42. [DOI:10.1016/j.jclinepi.2006.03.012] [PMID]

[37] Reio Jr TG, Shuck B. Exploratory factor analysis: Implications for theory, research, and practice. Advances in Developing Human Resources. 2015; 17(1):12-25. [DOI:10.1177/1523422314559804]

[38] Antony MM, Barlow DH, editors. Handbook of assessment and treatment planning for psychological disorders. New York: Guilford Press; 2011. https://books.google.com/books?id=tgCS5i2oRPAC\&dq

[39] LoBiondo-Wood G, Haber J, Titler MG. Evidence-based practice for nursing and healthcare quality improvement. St. Louis: Elsevier; 2018. https://books.google.com/books?id=nklmDwAAQBAJ\&dq 